\title{
Experimental Diffraction from HERA to
}

the $\mathrm{LHC}$

\author{
M. Arneodo, M. Diehl, V. Khoze, P. Newman \\ Diffractive Summary at IVth HERA-LHC Workshop \\ 30 May 2008
}

- Forward Physics projects at the LHC

- Diffractive Parton Densities

- Absorption and Gap Survival

- Unintegrated gluon density

- Closing in on Central Exclusive Production
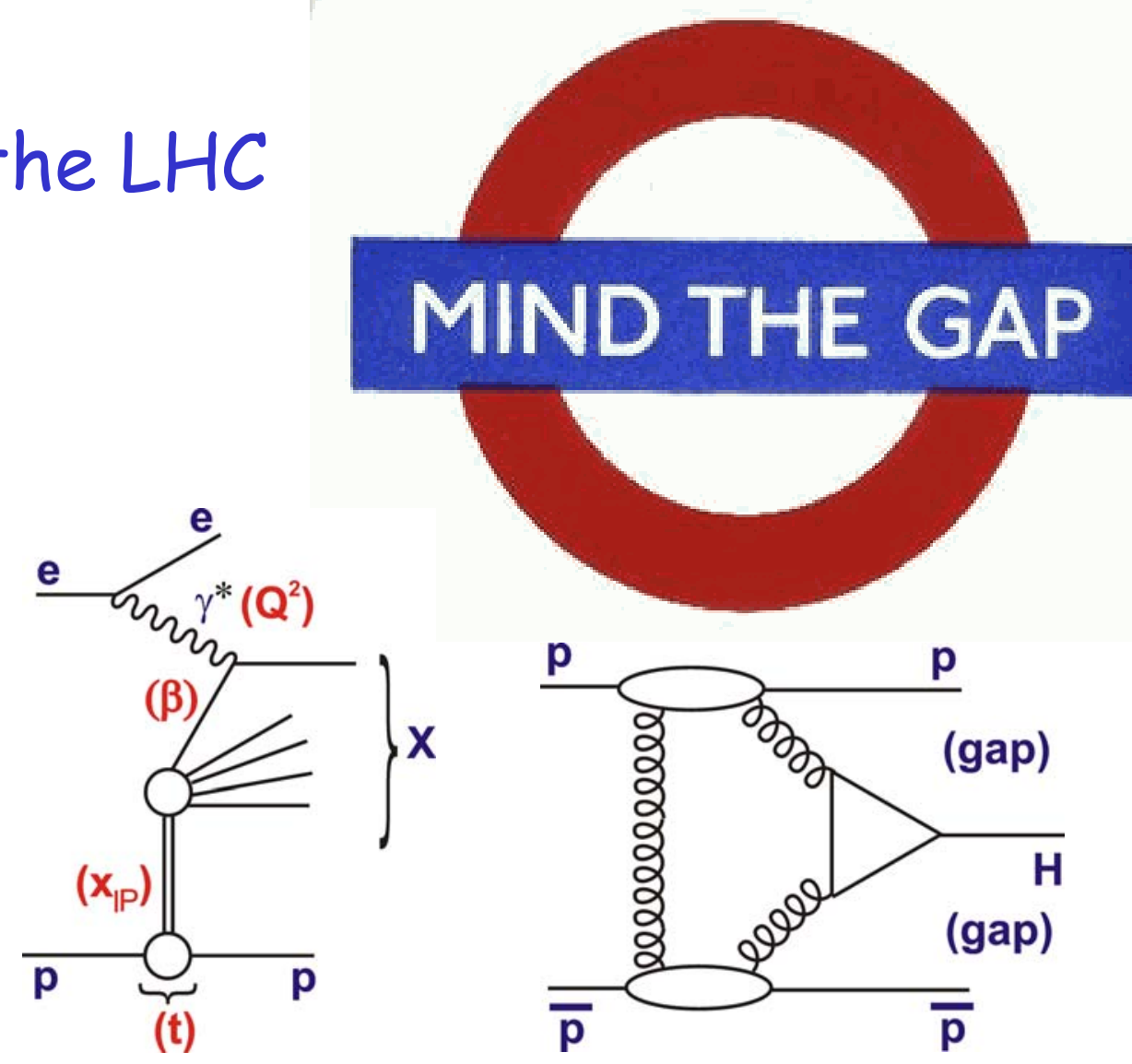


\section{List of Experimental Talks}

Review of Inclusive Diffraction at HERA

Review of Diffractive Dijets at HERA

Review of Leading Baryons at HERA

Review of HERA Vector Meson and DVCS Data

Exclusive Di-lepton and Di-photon Production at CDF-II Diffractive and Exclusive Dijets and $W / Z$ at CDF

Forward Physics at the LHC

Forward Physics with CMS

Diffractive and Forward Physics with Totem

Update on the AFP Project in ATLAS

The ALFA Detector: Status and Physics Programme

CASTOR Calorimeter: Physics and Status

Diffractive Physics in ALICE

Exclusive Dilepton Production at CMS

Single Diffractive W Production at CMS
Marta Ruspa

Alice Valkarova

Bill Schmidke

Pierre Marage

Jim Pinfold

Dino Goulianos

Peter Bussey

Samim Ehran

Ken Oesterberg

Christophe Royon

Karl-Heinz Hiller

Kerstin Borras

Rainer Schicker

Jonathan Hollar

Antonio Vilela-Pereira

- Short \& limited summary ... apologies for the many omissions

- Focus on developments in last year and open points 
LHC Forward Instrumentation

(many talks)
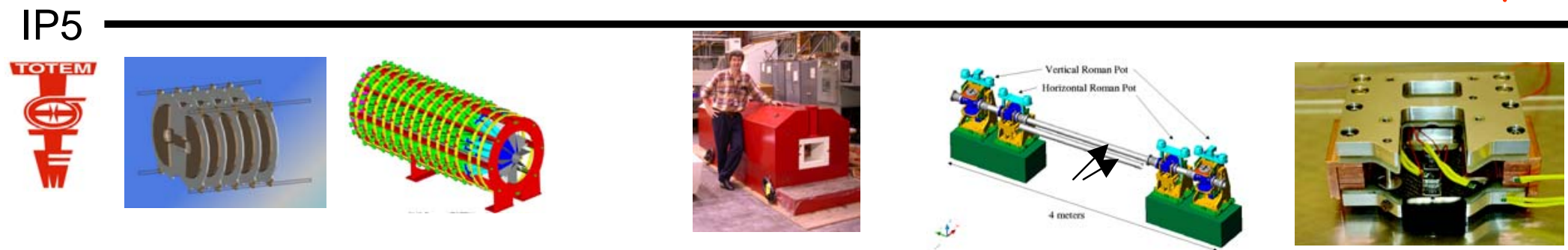

TOTEM-T2 CASTOR

$\mathrm{ZDC} / \mathrm{FwdCal}$

TOTEM-RP

FP420

$14 m$

$16 m$

$140 m$

147-(180)-220m

$420 m$

LUCID

ZDC

ALFA/FP220

FP420
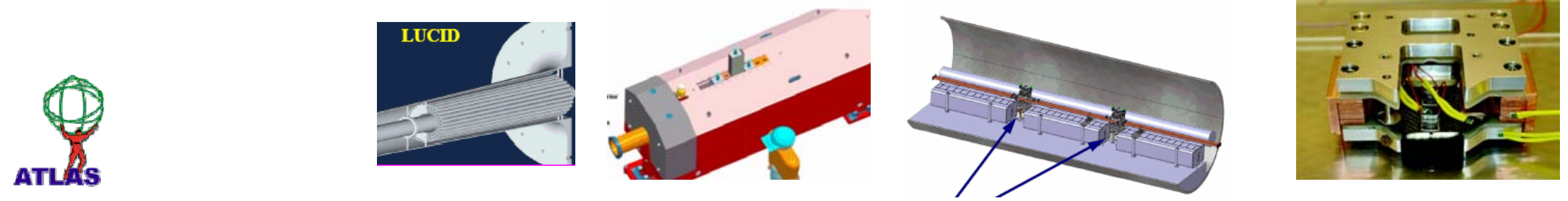

IP1

Impressive array of forward physics projects, providing high rapidity tracking / calorimetry and proton spectroscopy ...

... the best instrumented forward beam-lines ever! 


\section{LHC Proton Spectrometry}

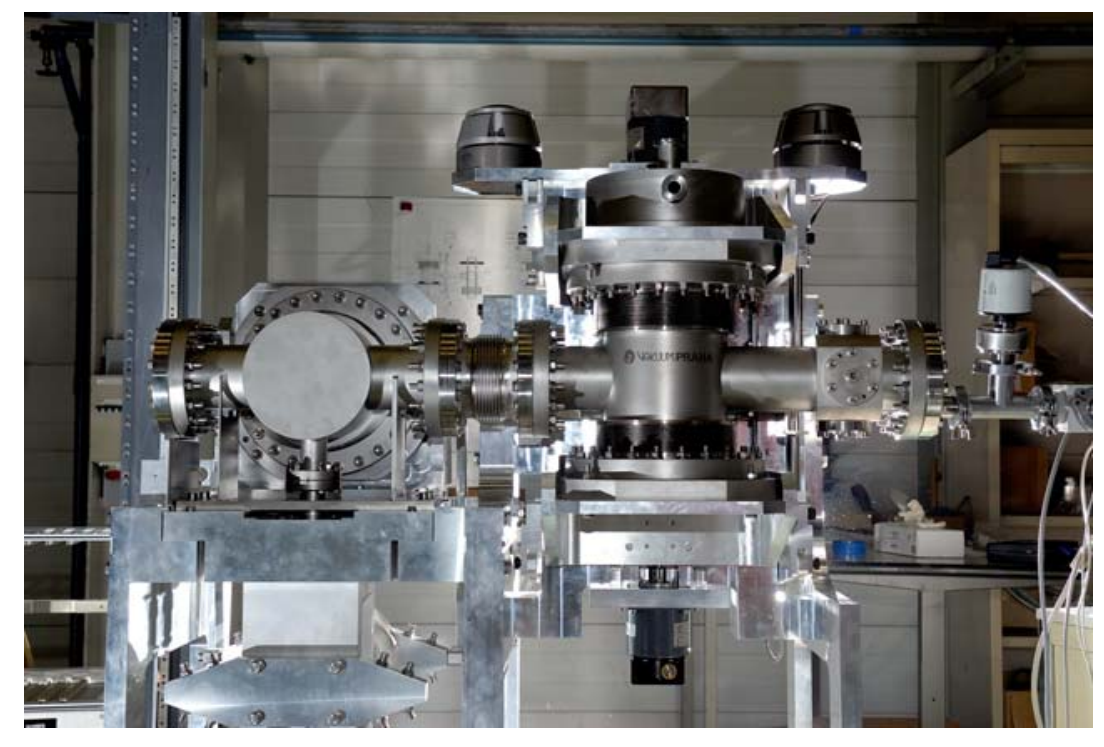

Pots up to $220 \mathrm{~m}$

TOTEM pots at $150 \mathrm{~m}$ and $220 \mathrm{~m}$ fully in place for 2009

FP220 proposed for installation in ATLAS >2009.

Pots at $420 \mathrm{~m}$

FP420 for ATLAS or CMS (installation >2009 if approved)

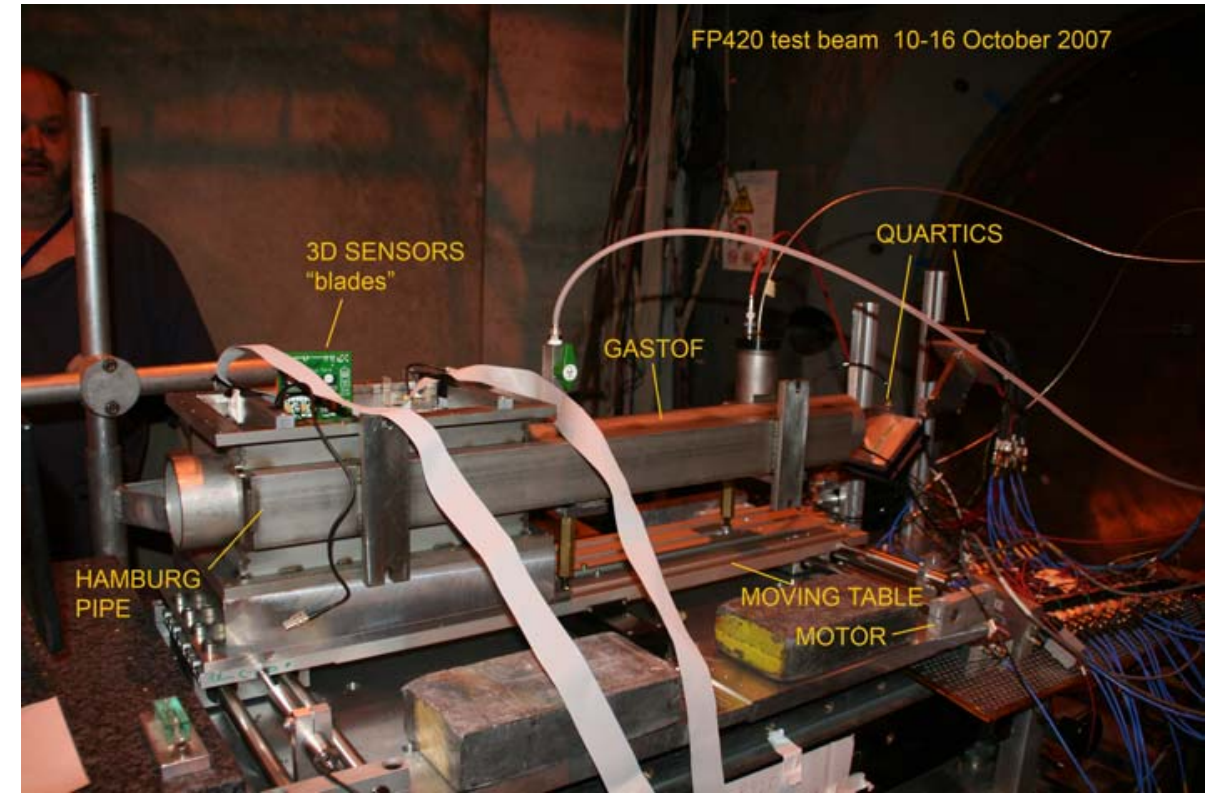


Proton Acceptance @ CMS / Totem

\section{$150 m+220 m$ Pots}

$0.02<\xi<0.2$ at $\beta^{\star}=0.5 \mathrm{~m}$ (high lumi optics)

Reaches lower $\xi$ at $\beta^{*}=90$ or 1540 m (lower lumi)

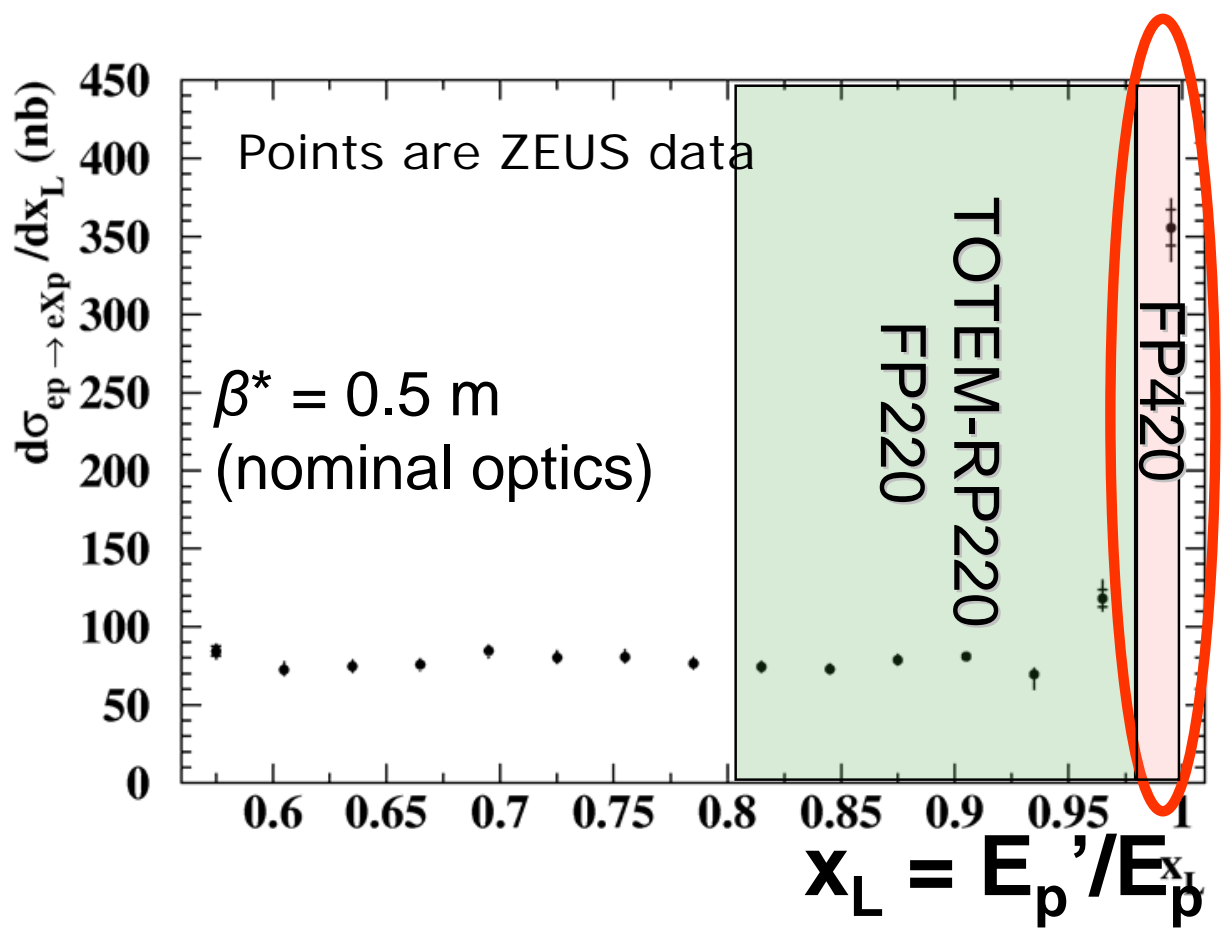

\section{$\log \xi=\log \left(1-E_{p}{ }^{\prime} / E_{p}\right)$}

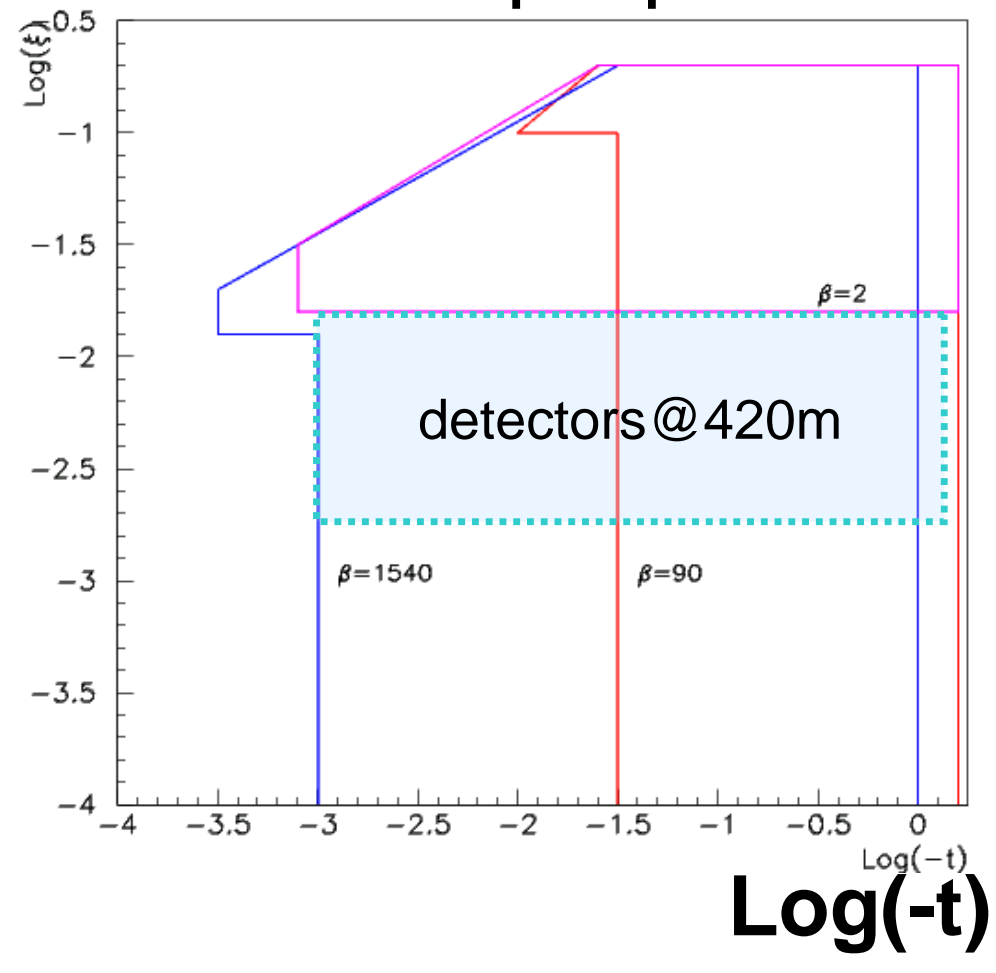

$420 \mathrm{~m}$ Pots

$0.002<\xi<0.02$ (high lumi optics) 


\section{Example Strategy: CMS + Totem + FP420}

Totem (tracking + pots) and CMS are highly complementary ... greater than the sum of their parts ...

$$
\text { essential to continue working together ... }
$$

\section{"Prospects for diffractive and forward physics at the LHC"} CERN/LHCC 2006-039/6-124, CMS Note 2007/002, TOTEM Note 06-5, Dec 2006

Low lumi: Large rapidity gap selection possible Proton tagging optional

... Soft diffraction, $\sigma_{\text {tot }}, \sigma_{\text {el }} .$.

... Some Hard diffraction / DPDFs

... Understanding pile-up

... $\gamma \gamma$ physics

High lumi: Pile-up ruins rapidity gap selection Proton tagging essential (220 \& $420 \mathrm{~m}$ )

... Diffractive Higgs and other exotica

... More hard diffraction / DPDFs

... $\gamma \gamma$ physics 


\section{Overcoming pile-up with Timing}

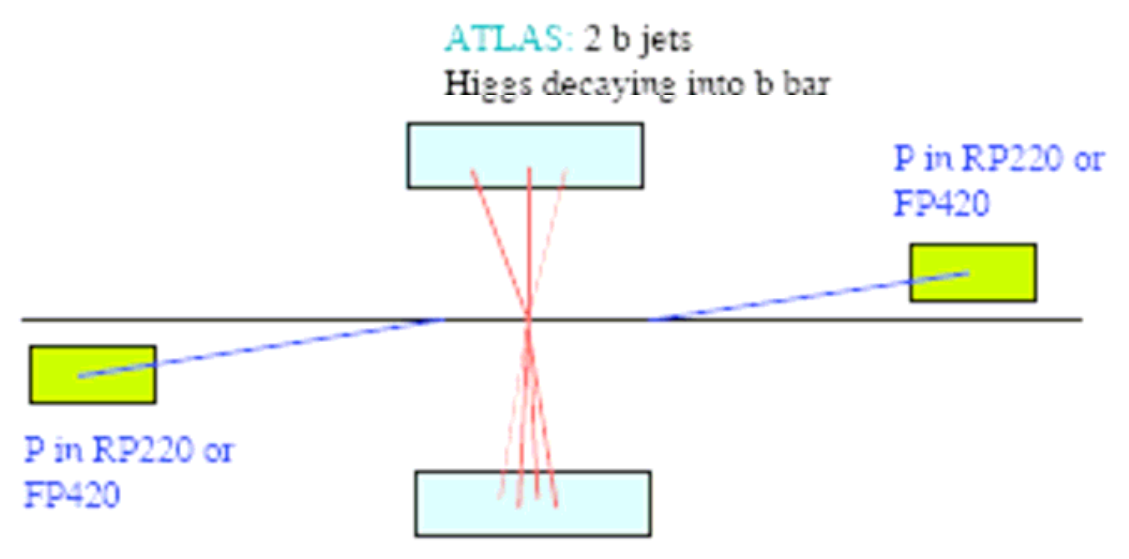

Proton spectrometer design incorporating precise (few ps) timing measurements, from which $z$ position of vertex can be reconstructed to a few $\mathrm{mm}$.

ATLAS: 2 b jets

Higes decaying into $\mathrm{b}$ bar

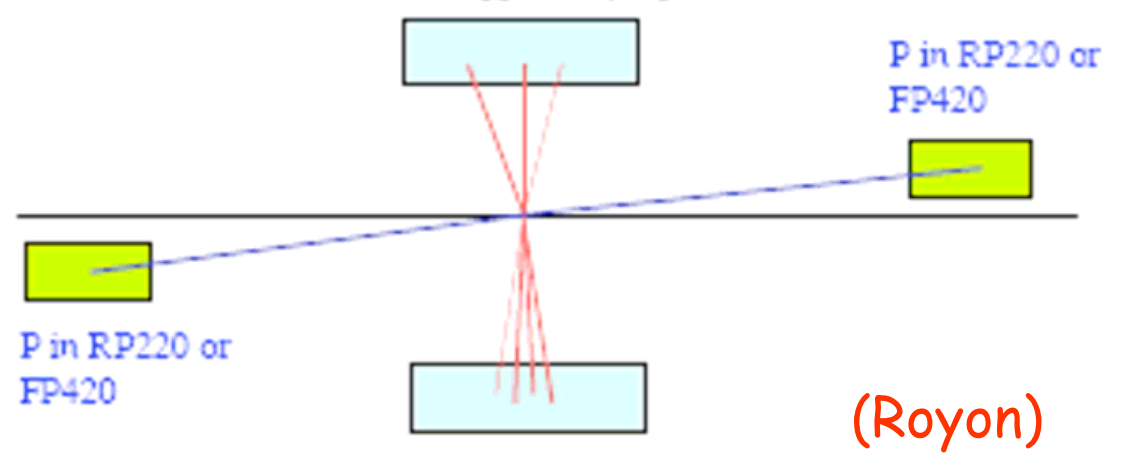

$\rightarrow$ Correlating leading protons with hard interaction in central detector in presence of (many) soft interactions 


\section{Diffraction at ALICE}

(Schicker)
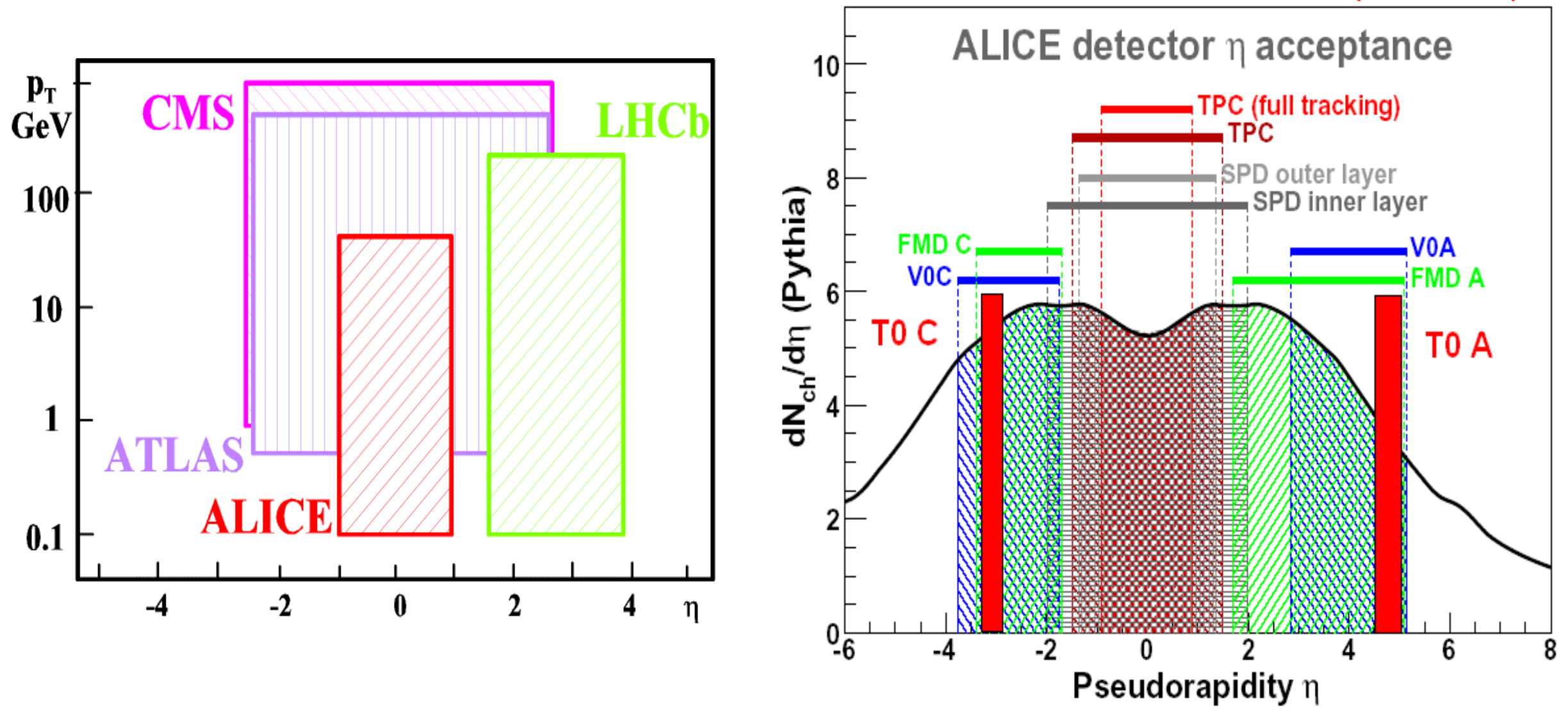

- (Uniquely?) capable of triggering on and detecting central tracks at very low pt.

- Together with $1^{\text {st }}$ level 'gap' trigger using scintilators and silicon, sensitive to 'soft' and low $p_{+}$diffractive processes

... e.g. $\gamma$ todderon $\rightarrow \mathrm{J} / \Psi$ searches 


\section{Diffraction at the LHC}

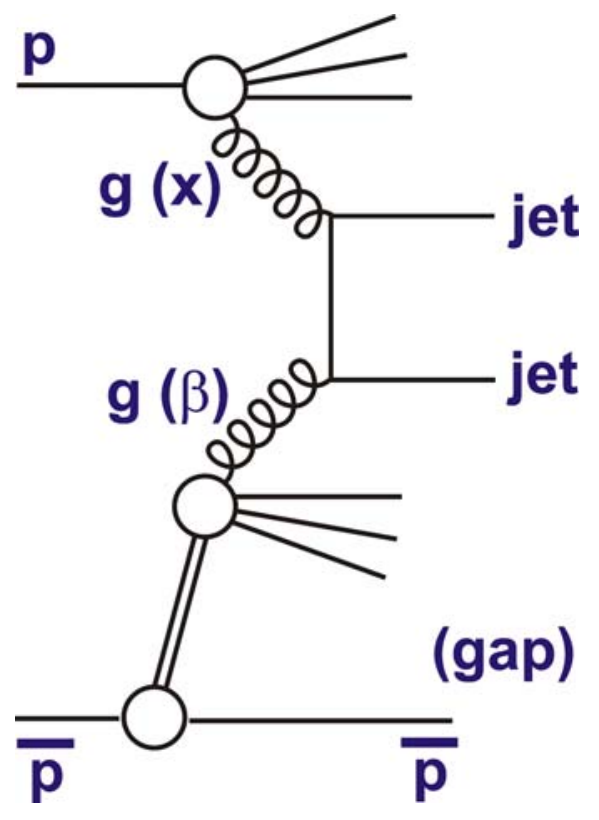

Opportunity to study Single and Double Diffraction with and without hard scales (jet, heavy flavours, W, Z).

$\rightarrow$ Depend on DPDFs from HERA

$\rightarrow$ Also on gap survival factors!
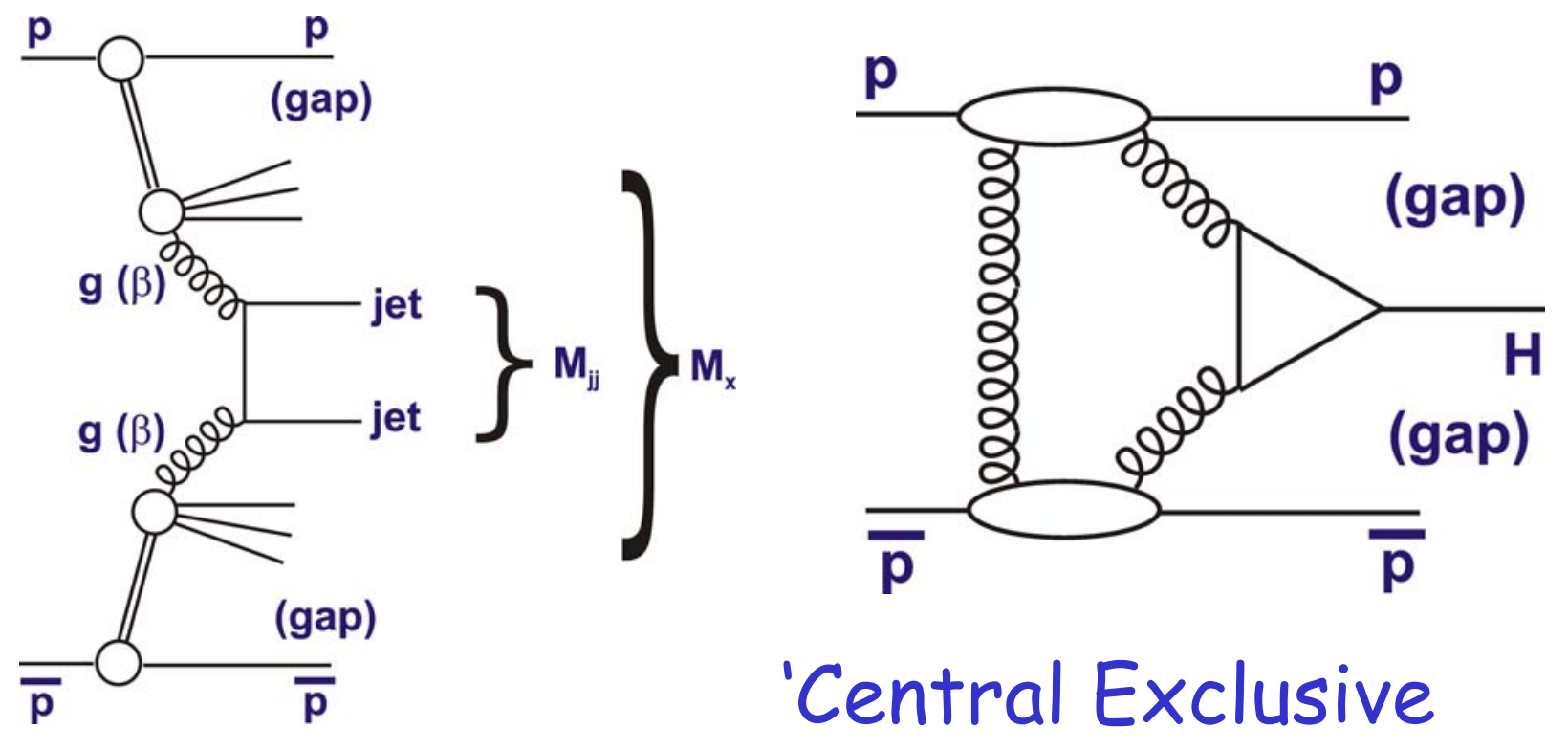

'Central Exclusive Production'

- DPDFs for backgd

- Unintegrated

gluon $\rightarrow \mathrm{J} / \Psi / \mathrm{Y}$

- Gap survival models (KKMR, GLM ...)

\section{... lots of possible input from HERA!}




\section{Inclusive ep Diffraction}

Senisitive to Difffractive

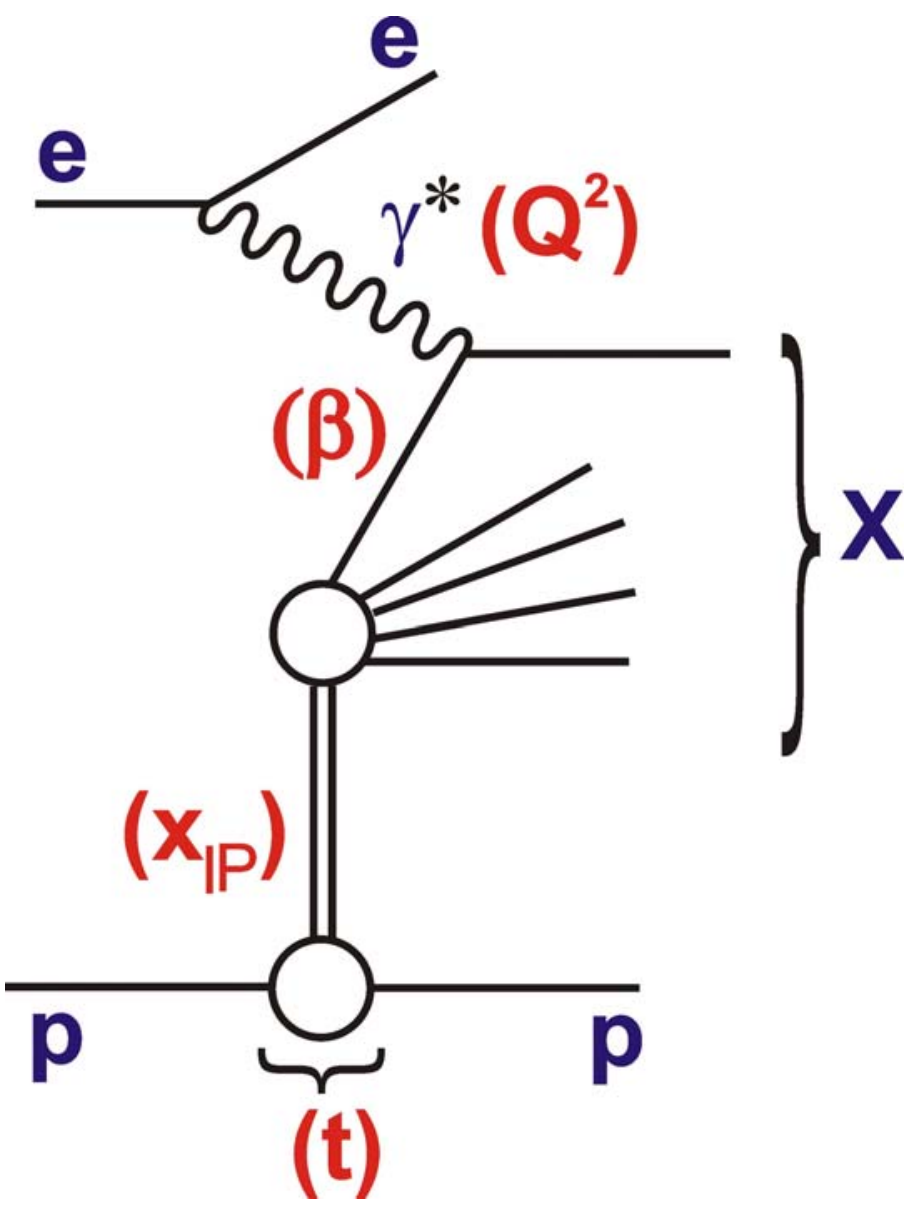

Parton Densities (DPDFs) and $t$ dependences

(See also Fracture Functions)

(Ceccopieri, Trentadue)

ZEUS and $\mathrm{H} 1$ both published highest precision measurements of 3 (or 4)-fold differential cross sections in past 2-3 years:

$$
\sigma_{r}^{D}\left(x_{I P}, \beta, Q^{2}\right)
$$

Work in HERA-LHC framework on level of agreement between collaborations and between different selection methods 


\section{Comparison of ZEUS v H1 Proton Tagged Data}

\section{ZEUS}

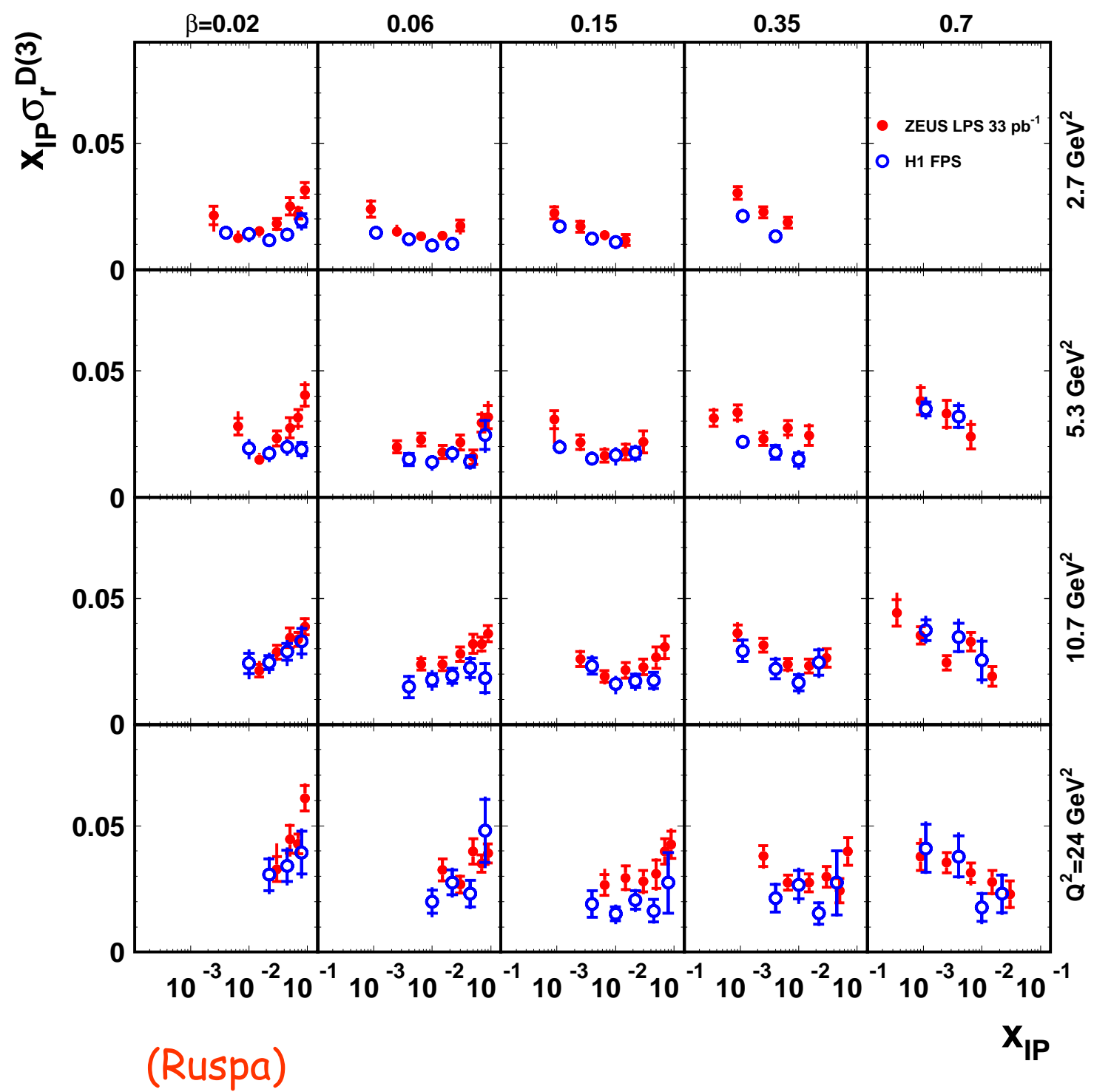

The cleanest

possible comparison in principle....

... good agreement within (large) normalisation uncertainties ( 10\% for each experiment)

Also very good agreement on low mass proton diss'n e.g. from LRG / FPS 


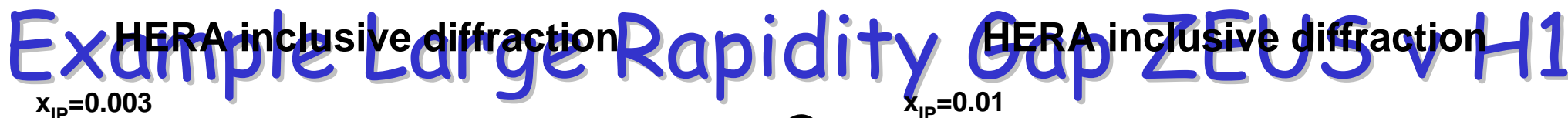
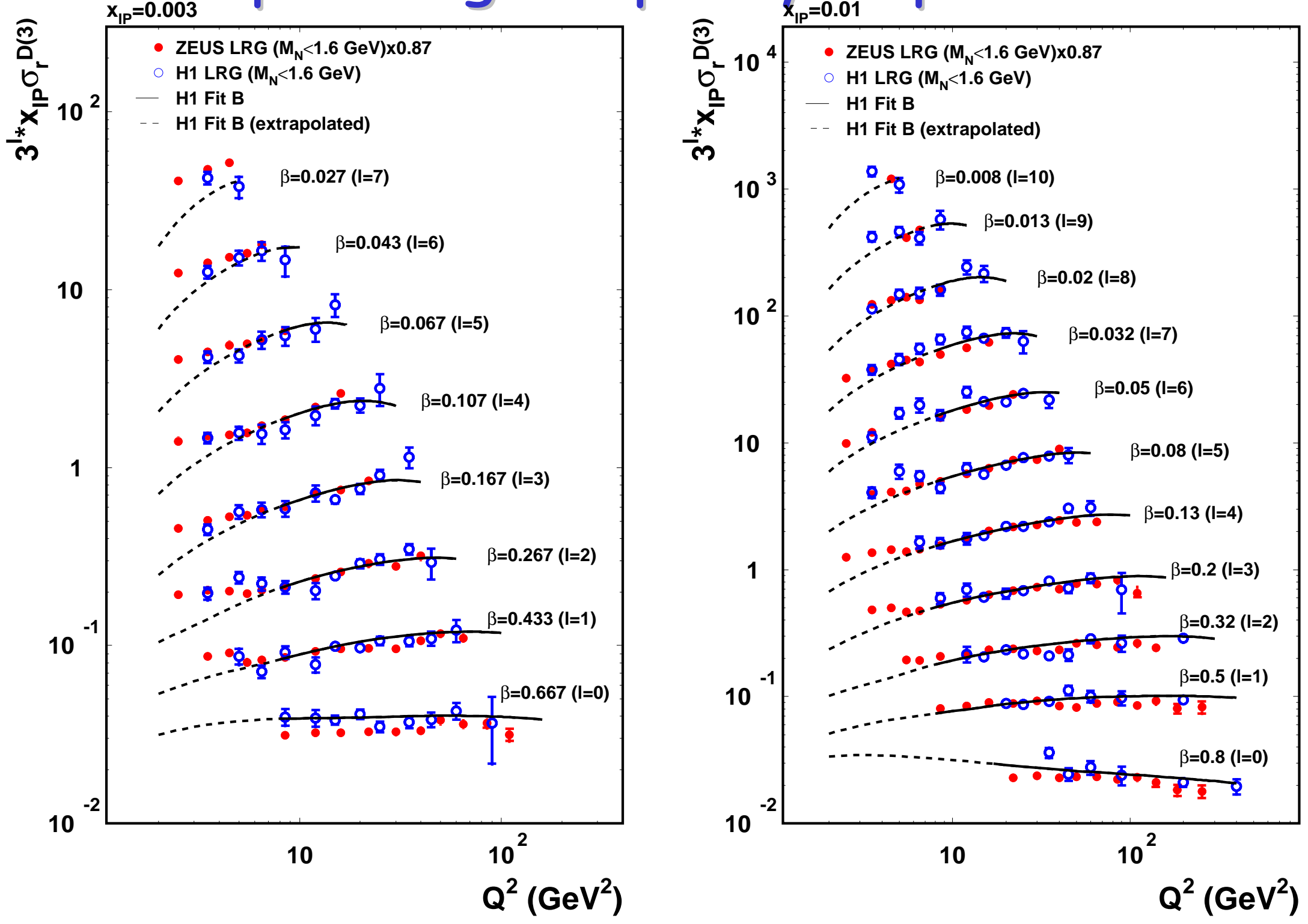

(Ruspa)

Very good agreement through most of phase space ... 


\section{Diffractive Parton Densities from $\sigma_{r}{ }^{D}$}

- $\sigma_{\mathrm{r}} \mathrm{D}$ gives quark density to $\sim 5 \%$, gluon to $\sim 15 \%$ at low $z$, increasing rapidly as $z \rightarrow 1$.

- Well tested in DIS final states (jets / charm)

... but high z very important!

- Background to CEP!

- 'Direct' IP (eg MRW)

- Including jet and charm data much improves high z
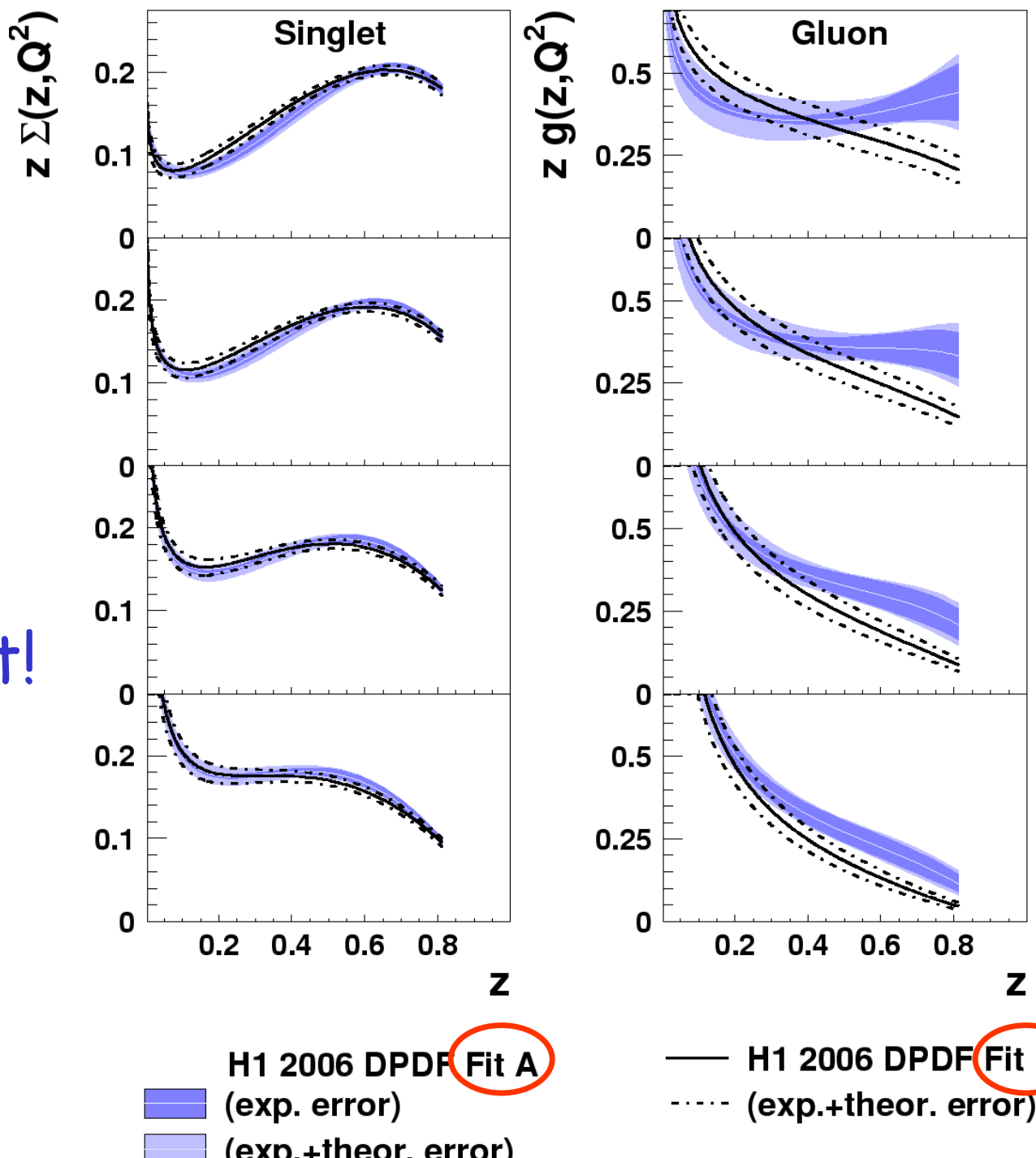

$\mathbf{Q}^{2}$

8.5

20

90

800

— H1 2006 DPDFFit B

-... (exp.+theor. error)

Next steps: Similar ZEUS fit

: More data comparisons and joint (' global'?) fit 


\section{t Dependence of $\sigma_{r}^{D}$ from LPS / FPS}

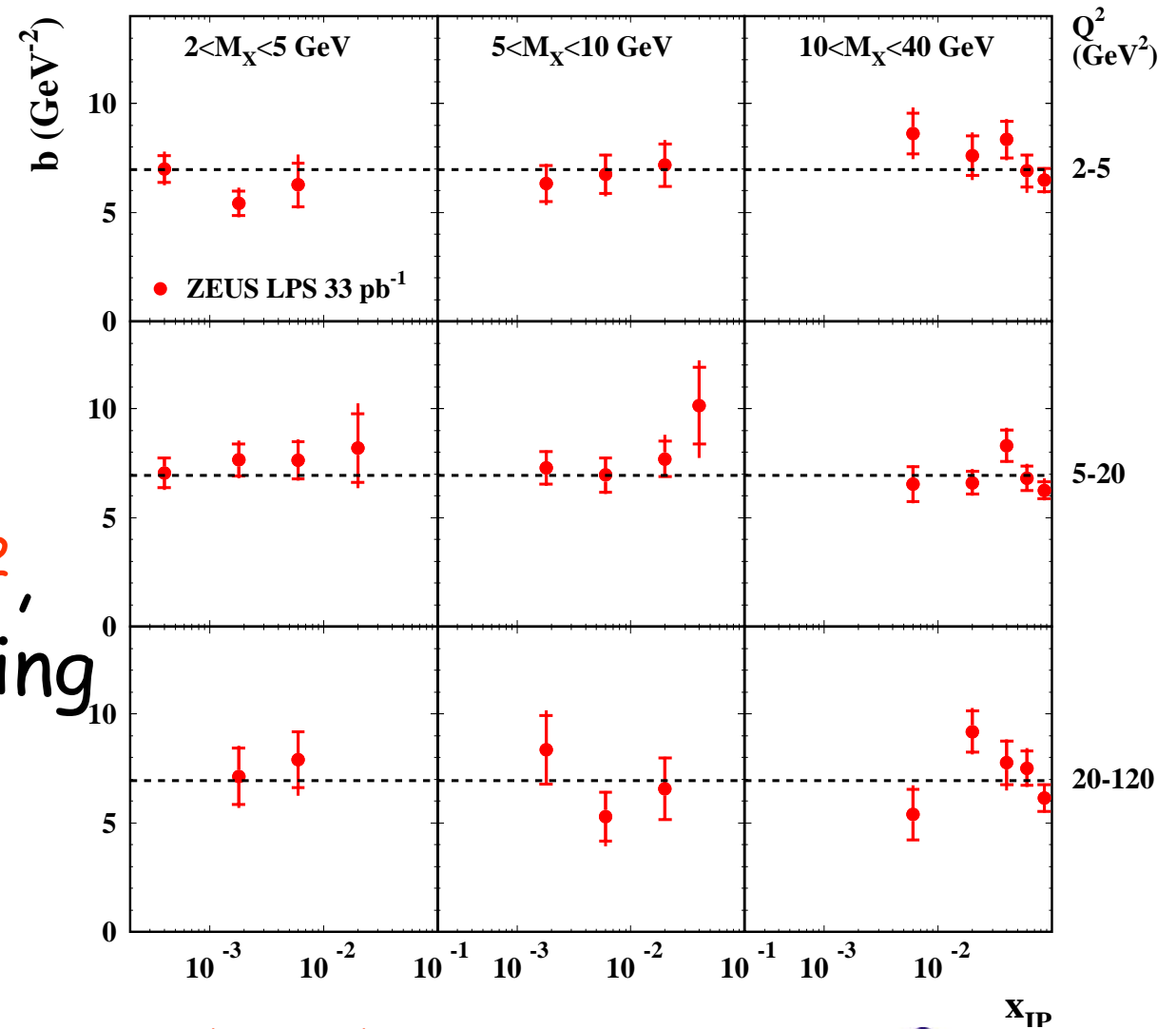

Very good ZEUS v H1 agreement on effective pomeron trajectory:

$\alpha_{I P}(0)=1.11-1.12 \quad$ (various extractions)

$\alpha_{\text {IP }}^{\prime}=-0.01 \pm 0.06\left(\right.$ stat) $+0.04-0.08$ (syst) $\mathrm{GeV}^{-2}$

(ZEUS LPS)

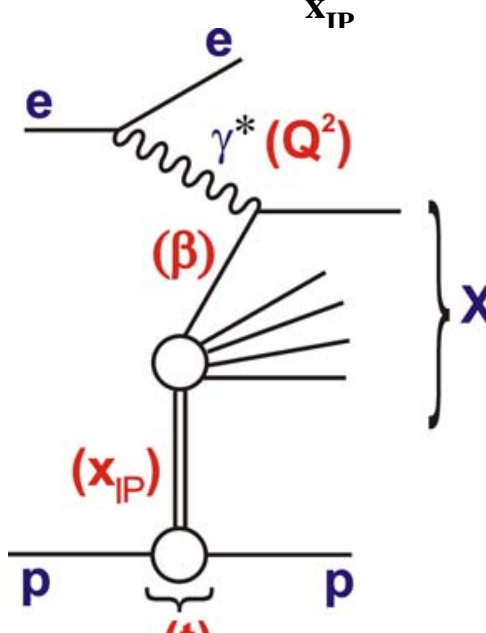

(t)

... not soft IP $\rightarrow$ multi-IP exchange / absorption in the game! 


\section{What is a soft pomeron anyway?}

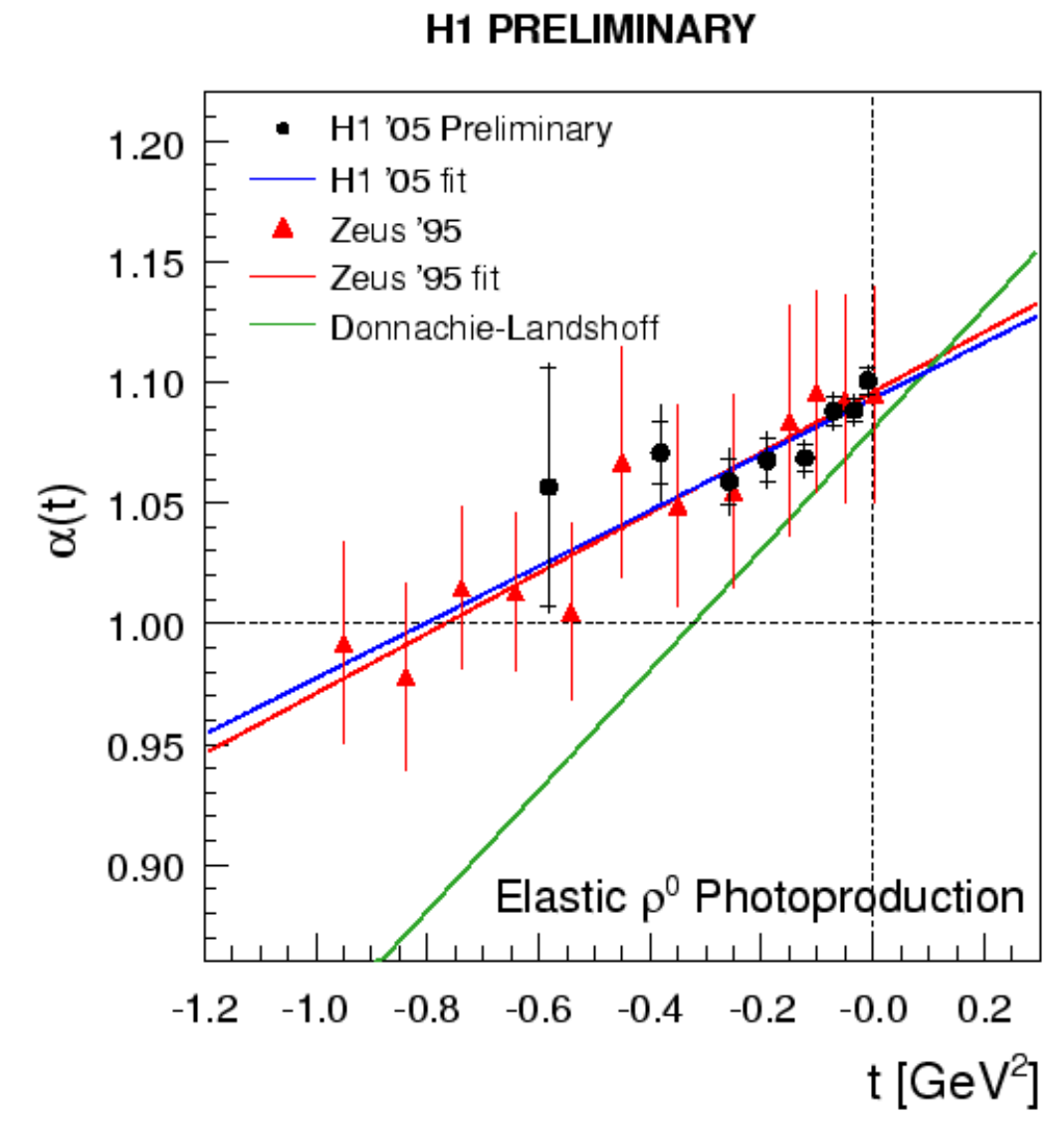

H1

$$
\alpha_{I P}(t)=[1.093 \pm 0.008]+[0.116 \pm 0.049] t
$$

ZEUS

$$
\alpha_{I P}(t)=[1.096 \pm 0.021]+[0.125 \pm 0.038] t
$$

'Soft'
The 'soft' pomeron in photoproduction at HERA is not as soft as we expected from pp

From $\gamma p \rightarrow \rho p$

(ZEUS $\phi$ similar)

(Marage)
There is NO universal IP even in soft physics!

$$
\alpha_{I P}(t)=[1.085]+[0.25] t
$$

Can be explained by different absorptive corrections? 


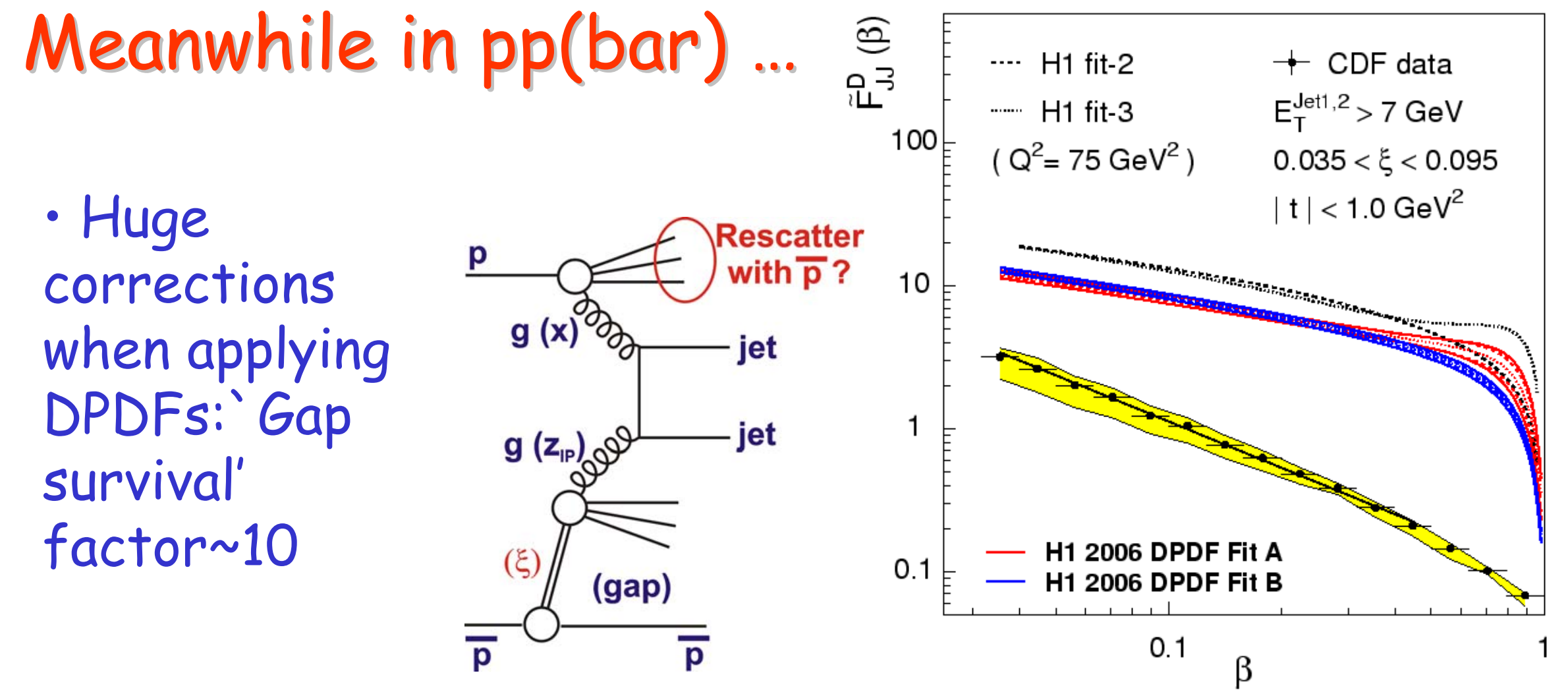

\section{... rp as a Control Expt?}

Most models predict gap survival probability ... $=1$ (direct) $<1$ (resolved ... e.g. Kaidalov, Khoze, Martin. Ryskin $\rightarrow \sim 0.34$ )
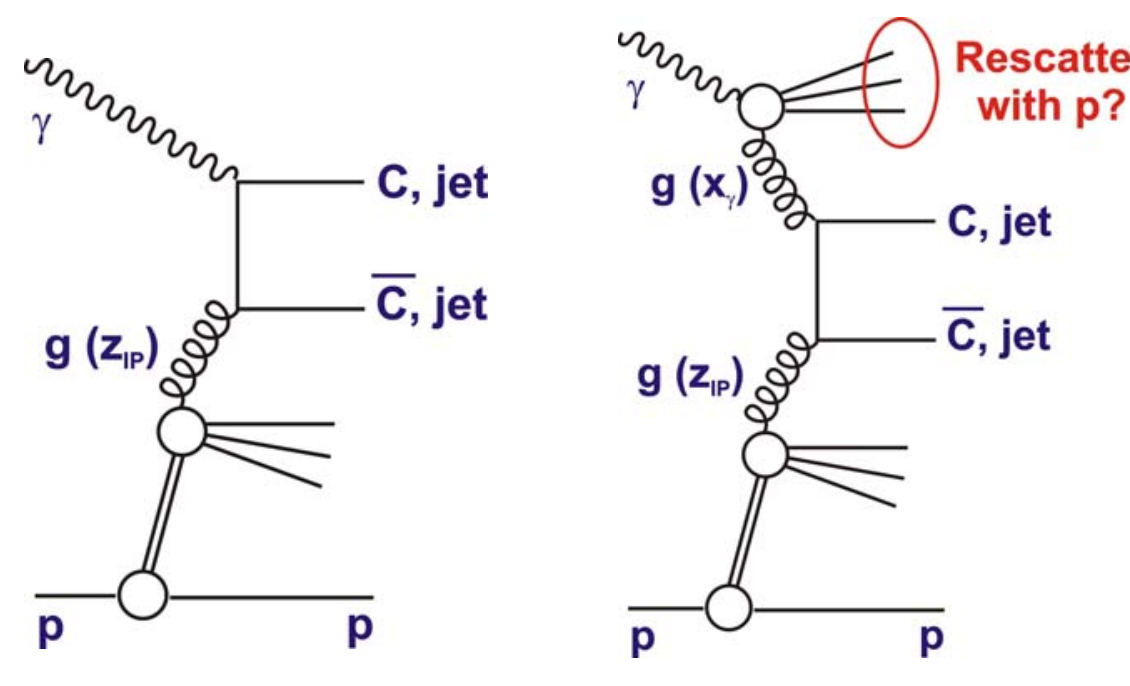


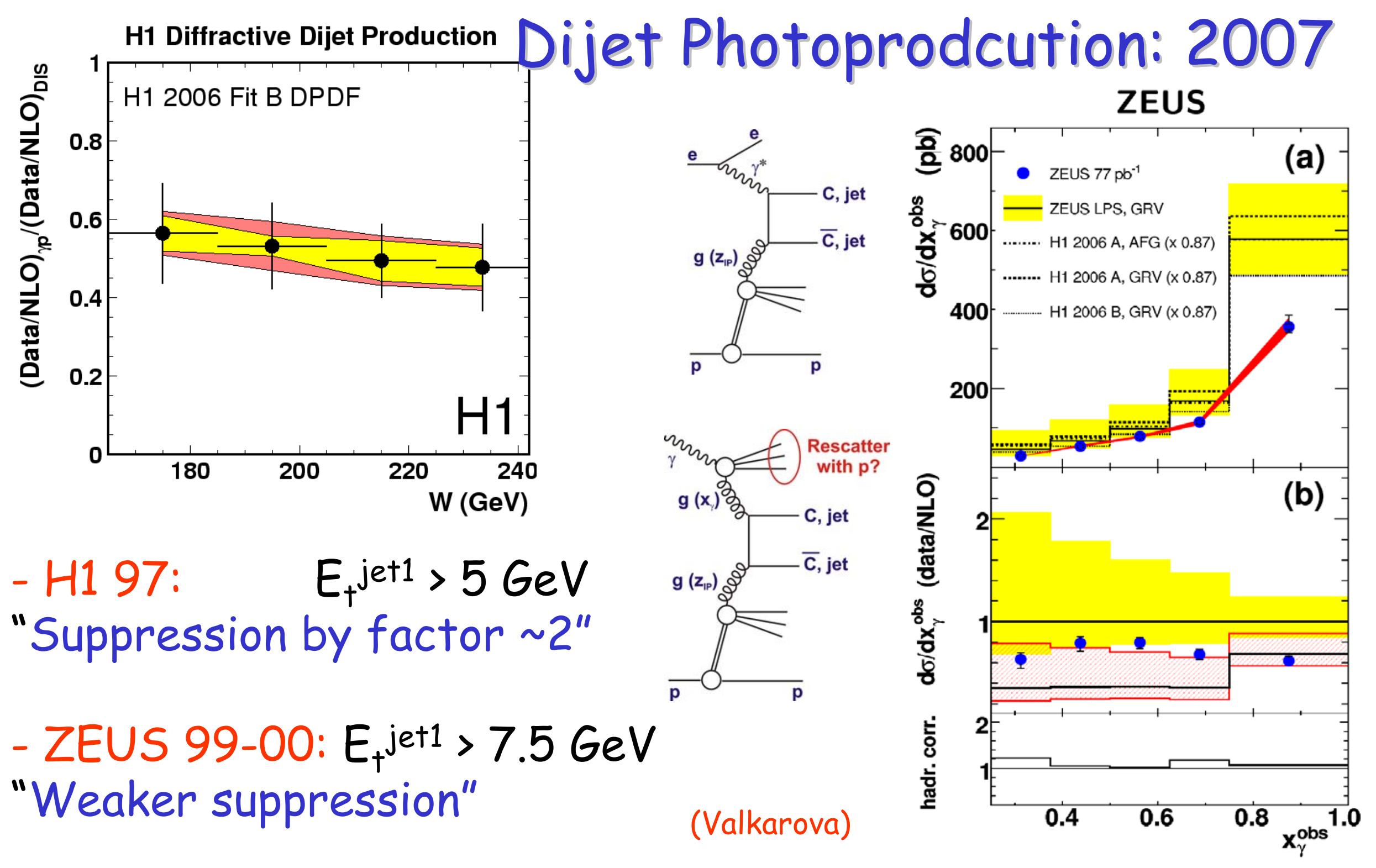

- Neither collaboration sees difference between resolved and direct regions, in contrast to theoretical expectations! 


\section{Recent Developments}

"H1 - ZEUS difference due to different $E_{T}$ ?" (DIS07, ZEUS)

H1 Diffractive Dijet Production

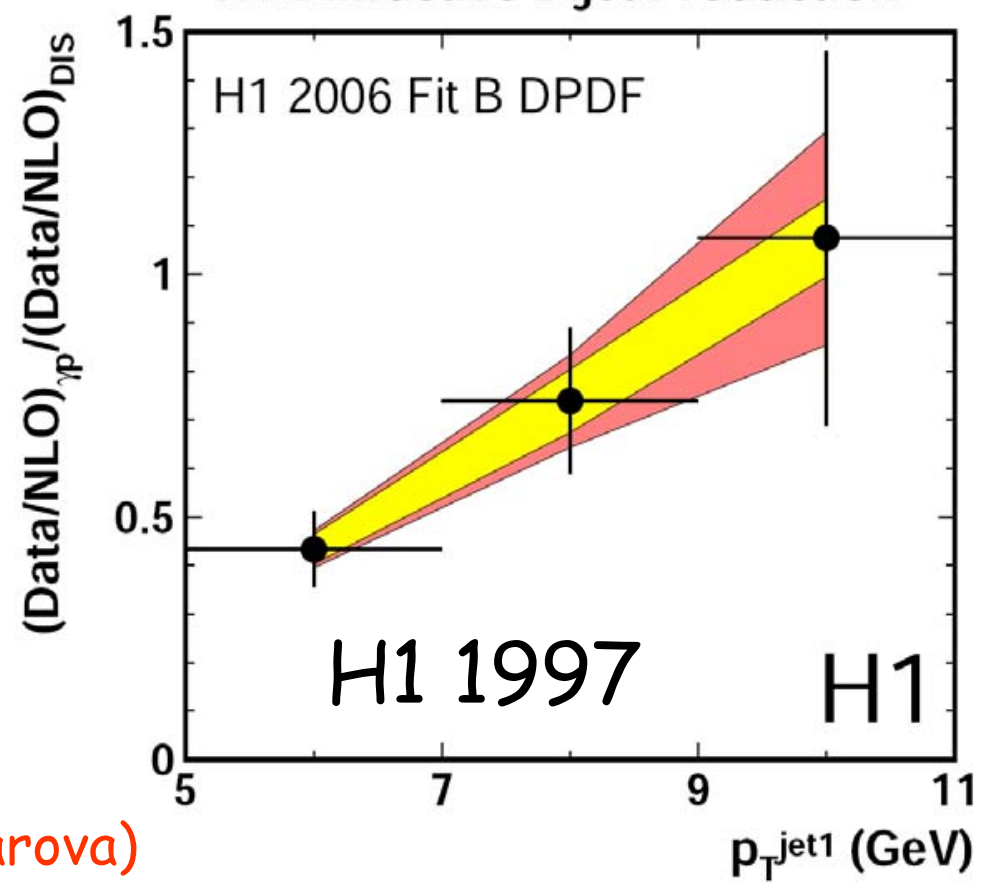

(ZEUS 9.9-00 data)

data

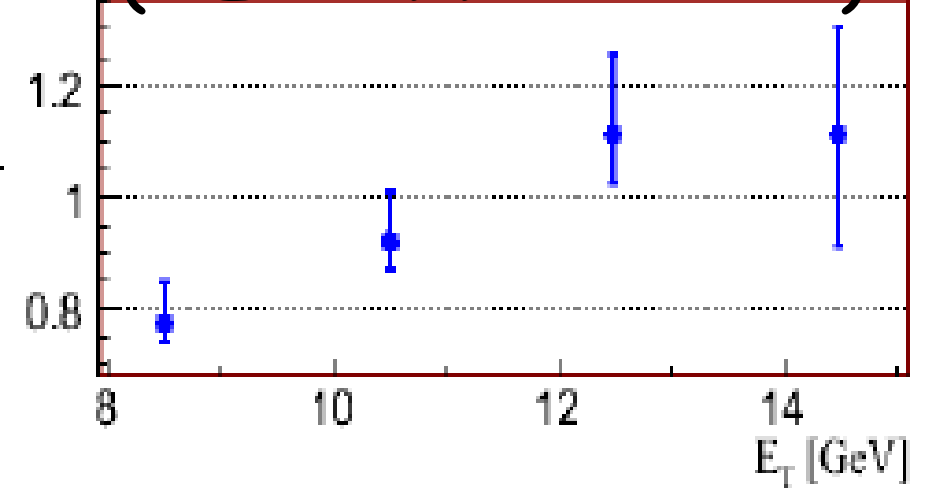

H1 PRELIMINARY

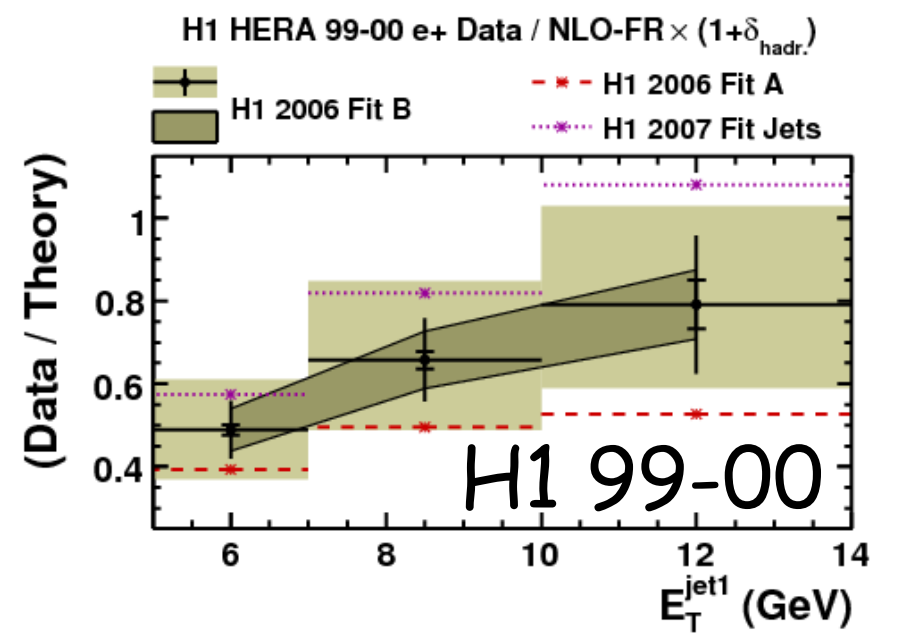

? Rescattering probability $\alpha$ Photon Size $r \alpha 1 / E_{T}$ ?

Understanding H1-ZEUS discrepancies is a success for the workshop, but probably tells us more about the high energy photon than it does about the $\mathrm{LHC}$ ! 


\section{Absorptive Corrections in Leading Neutrons}

- Total yield $0.6<x_{L}<0.9$ :

\section{ZEUS}

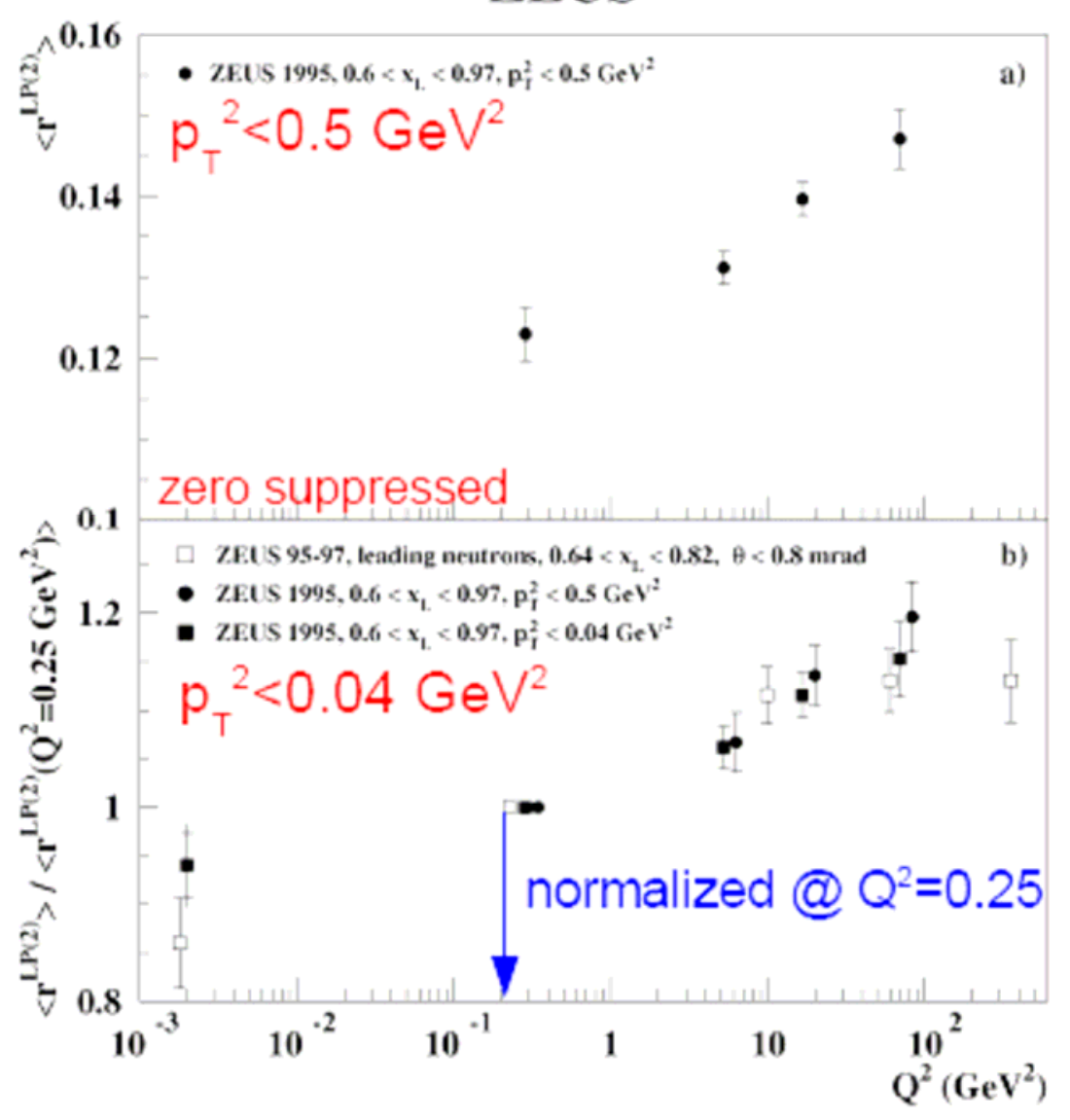

(Schmidke)

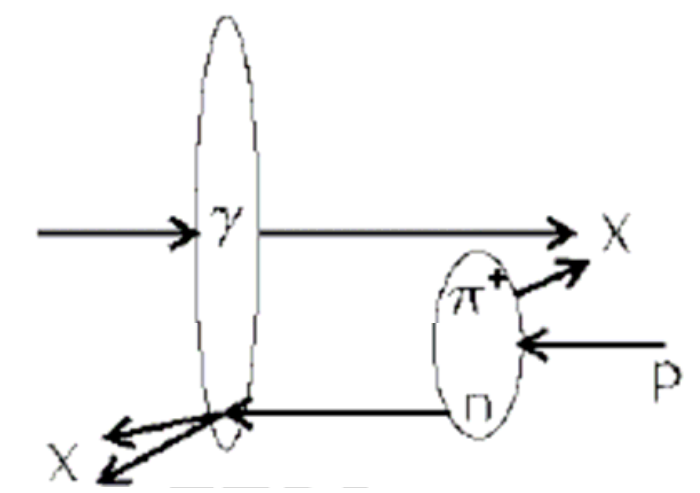

- Precise leading baryon data!

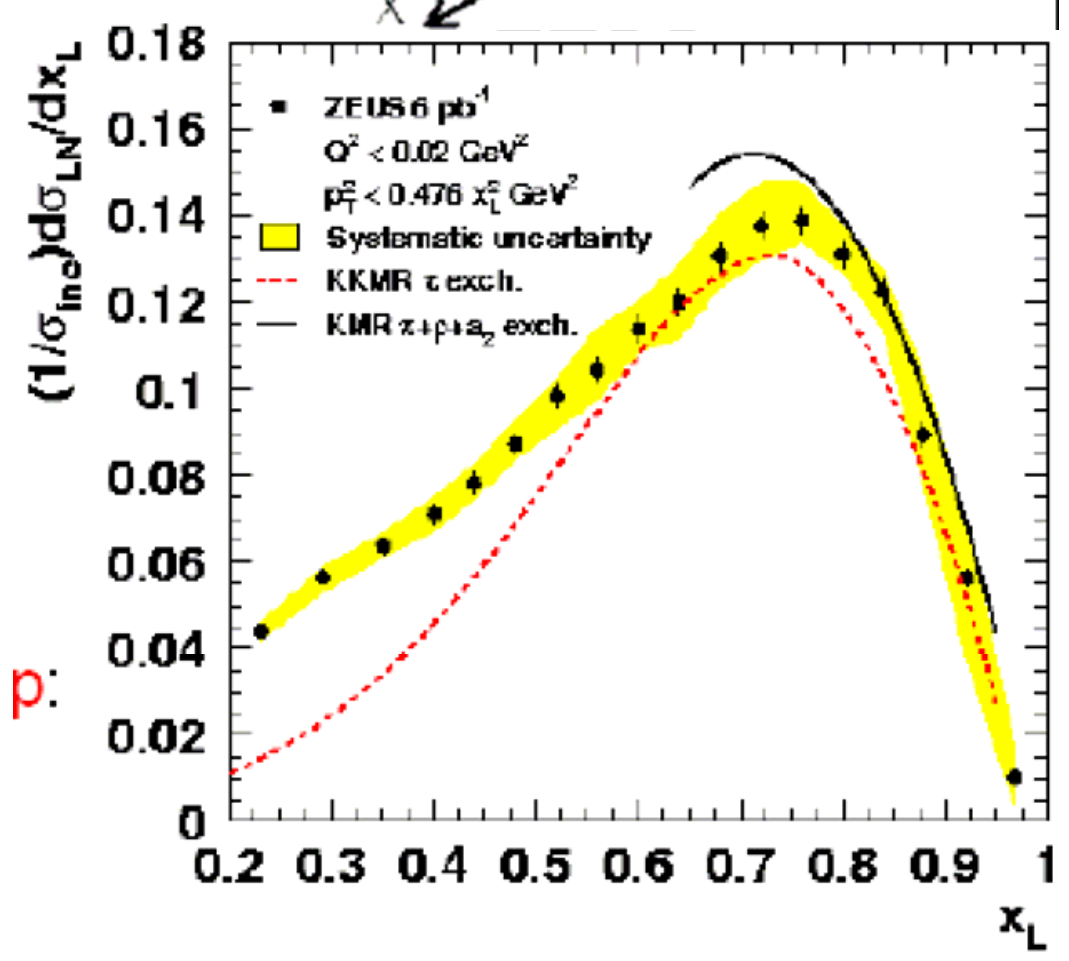

- LN yield decreases as $Q^{2}$ decreases, can be interpreted semi-quantitatively as absorptive effect. (Kaidalov et al) 


\section{Vector Mesons and DVCS at HERA}

Wealth of data (33 H1 + ZEUS papers, many more theory)

- Tests many pictures of

$\gamma\left({ }^{*}\right)$ p scattering ...

- most commonly,

scaterring colour dipoles from the proton

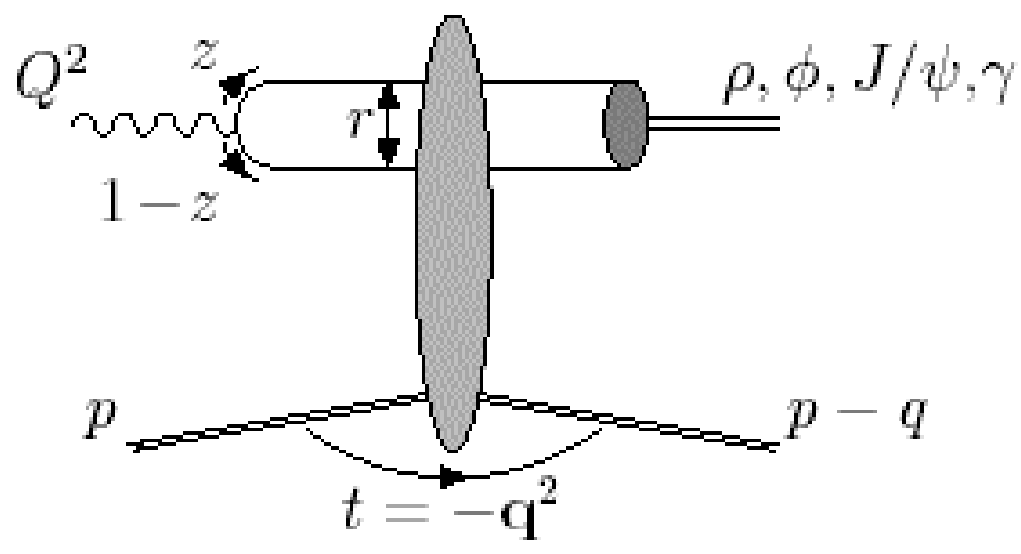

- Unique transverse / longitudinal $\gamma^{\star}$ separation

- Unique sensitivity to 'soft $\rightarrow$ hard' transition

- † measurements $\rightarrow$ transverse picture of proton

- Proton vertex factorisation tests e.g. from PD / EL

- Constraints on generalised gluon density (best with heavy VM) 


\section{Heavy VMs $\rightarrow$ Generalised Gluon Density}

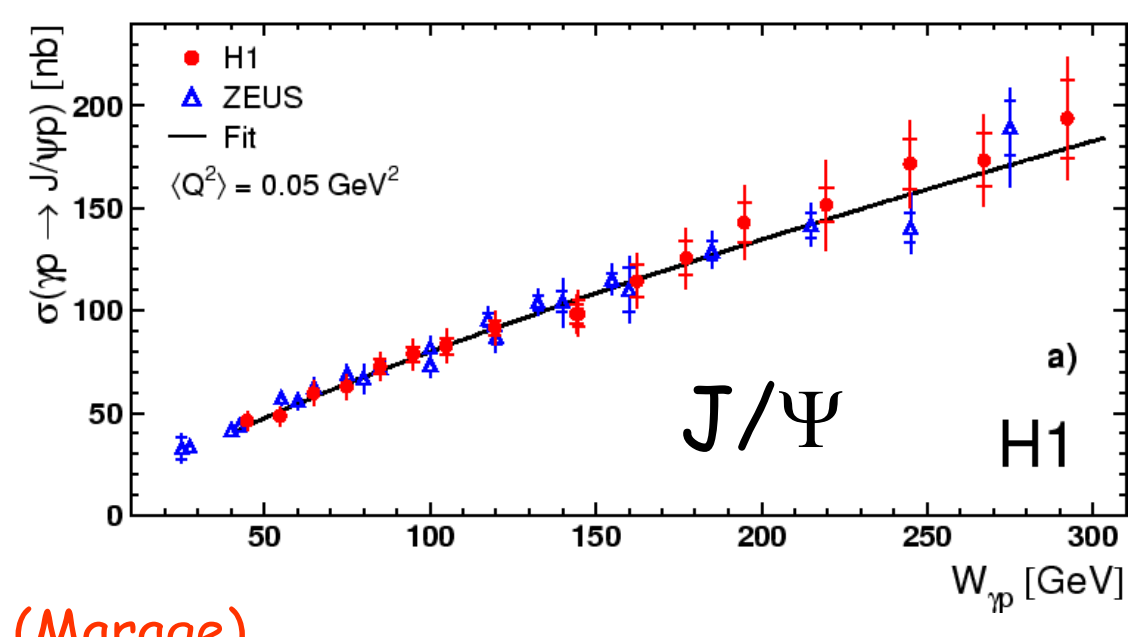

High precision $\mathrm{J} / \Psi$ data,

$\rightarrow$ Gluon (see e.g. Teubner et al.)

... Still statistics limited in (double differential) places

(Marage)

... every reason to analyse full HERA-II data

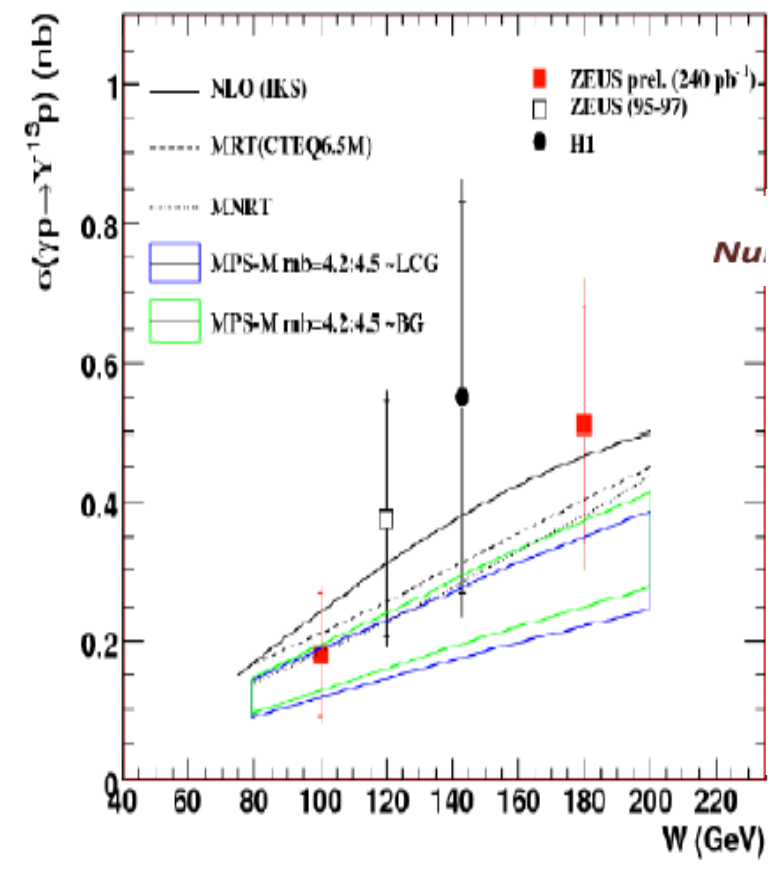

Even with

HERA-II

there will

not be big

Y Yields

At HERA

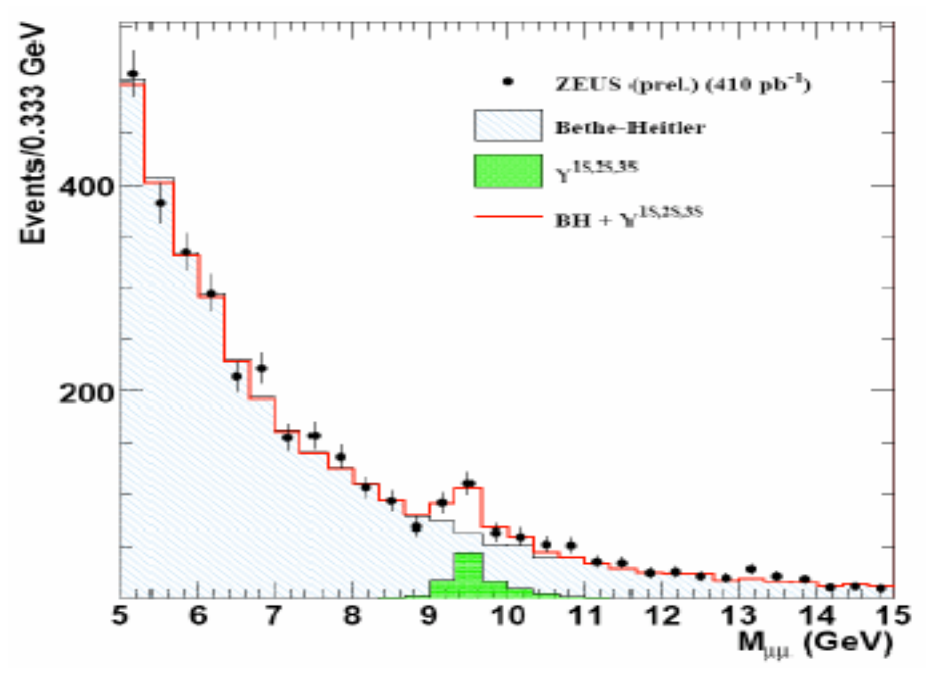

... but maybe Tevatron / LHC? 


\section{Heavy VM Photoproduction at Tevatron}

$\mathrm{p}$

CDF Exclusive ( $\gamma$ IP) Data

$\Delta \phi>120^{0}, \mathrm{p}_{\mathrm{T}}\left(\mu^{+}+\mu^{-}\right)<7 \mathrm{GeV} / \mathrm{c}$

o

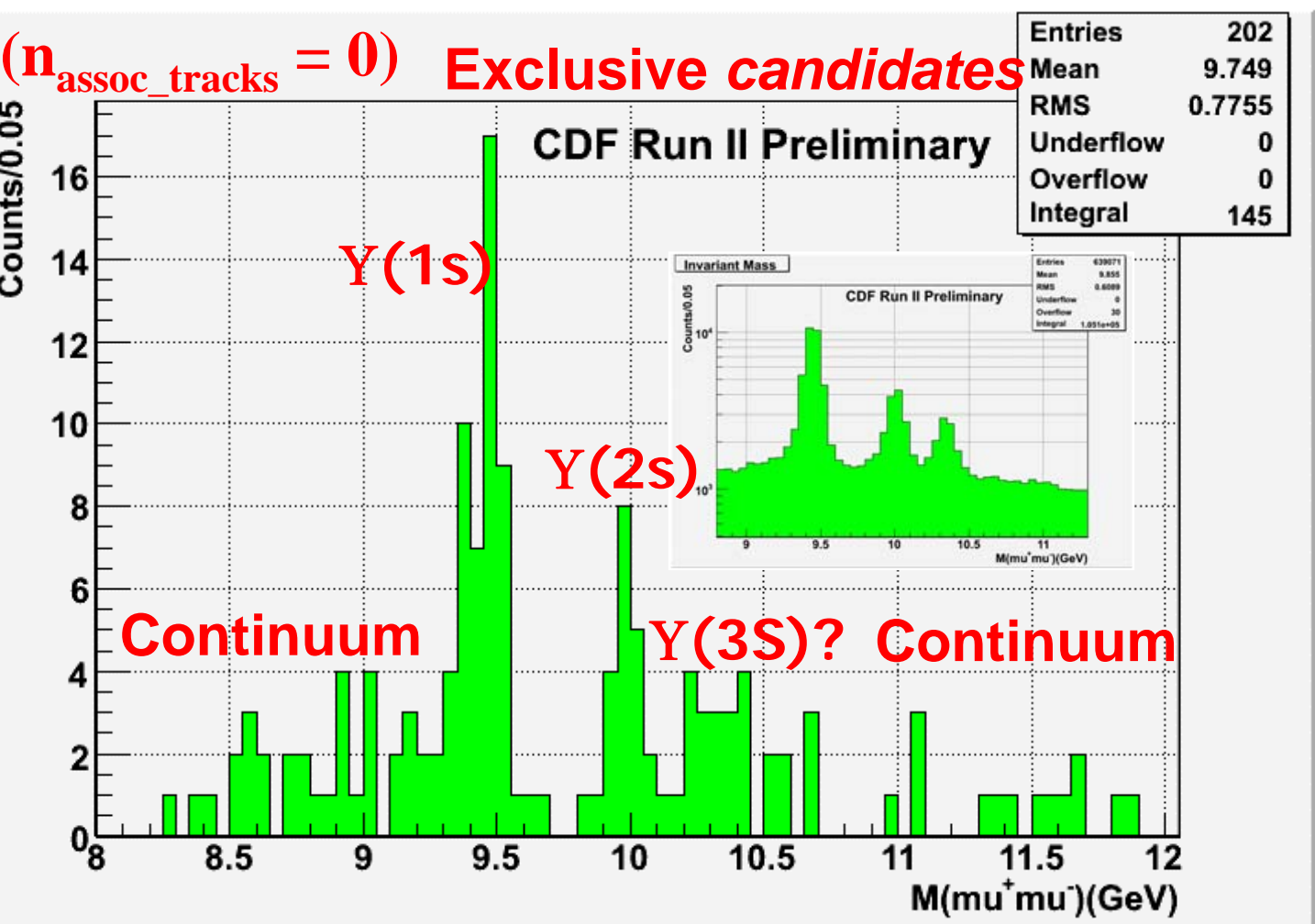

... potential to add further statistics ... 


\section{Final samples (100 $\left.\mathrm{pb}^{-1}\right)$}

- In MC, several hundred two-photon and Upsilon events pass the final selection in the dimuon channel

$709 \pm 27$ (stat) elastic events

$223 \pm 15$ (stat) \pm 42 (model) singly inelastic events

$636 \pm 25$ (stat) $\pm I 2 I$ (model) singly inelastic events, no ZDC/Castor

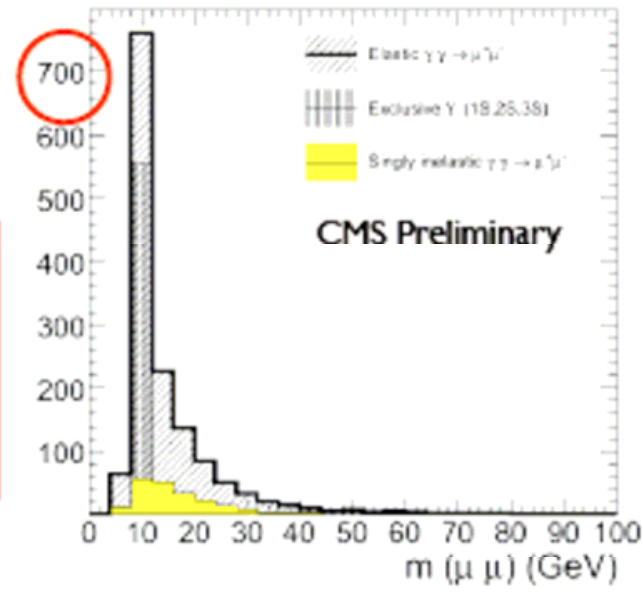

- Electron sample a factor of $\sim 10$ smaller due to higher trigger threshold, efficiency for low $\mathrm{E}_{\mathrm{T}}$ electron reconstruction

- No sensitivity to Upsilon region

$67 \pm 8$ (stat) elastic events

$3 \mathrm{I} \pm 6$ (stat) \pm 6 (model) singly inelastic events

$82 \pm 9$ (stat) \pm I5 (model) singly inelastic events, no ZDC/Castor

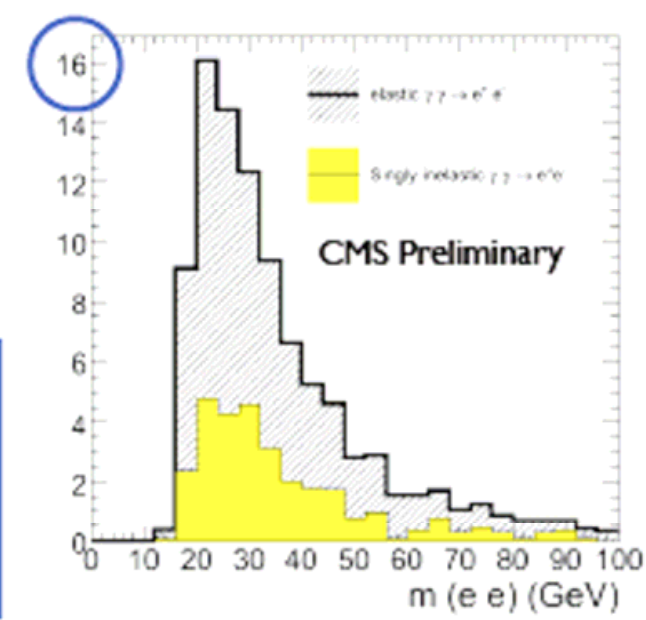

(Hollar) 
Exclusive Dijet Production at the Tevatron?

"DPE" dijets, plot $R_{\mathrm{jj}}=M_{\mathrm{jj}} / M_{x}$
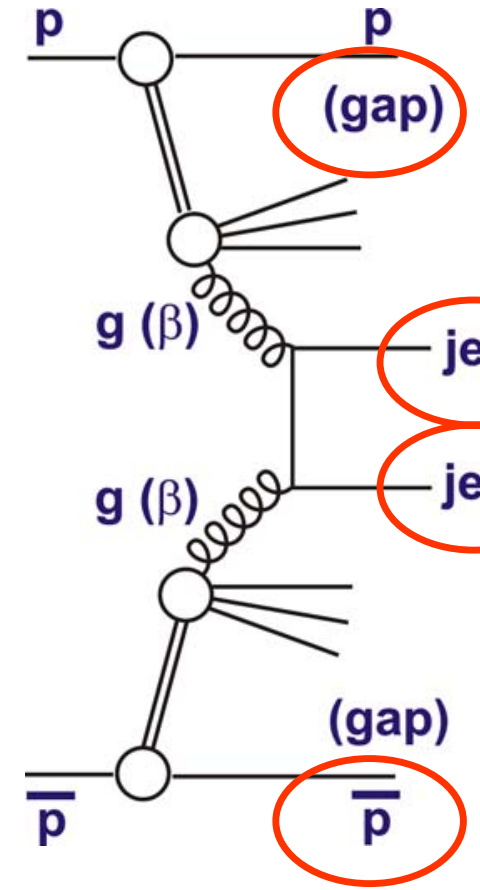

$R_{j j} \rightarrow 1$ for

exclusive

(complicated by hadronisn, higher order $Q C D \ldots)$

\section{CDF Run II |}

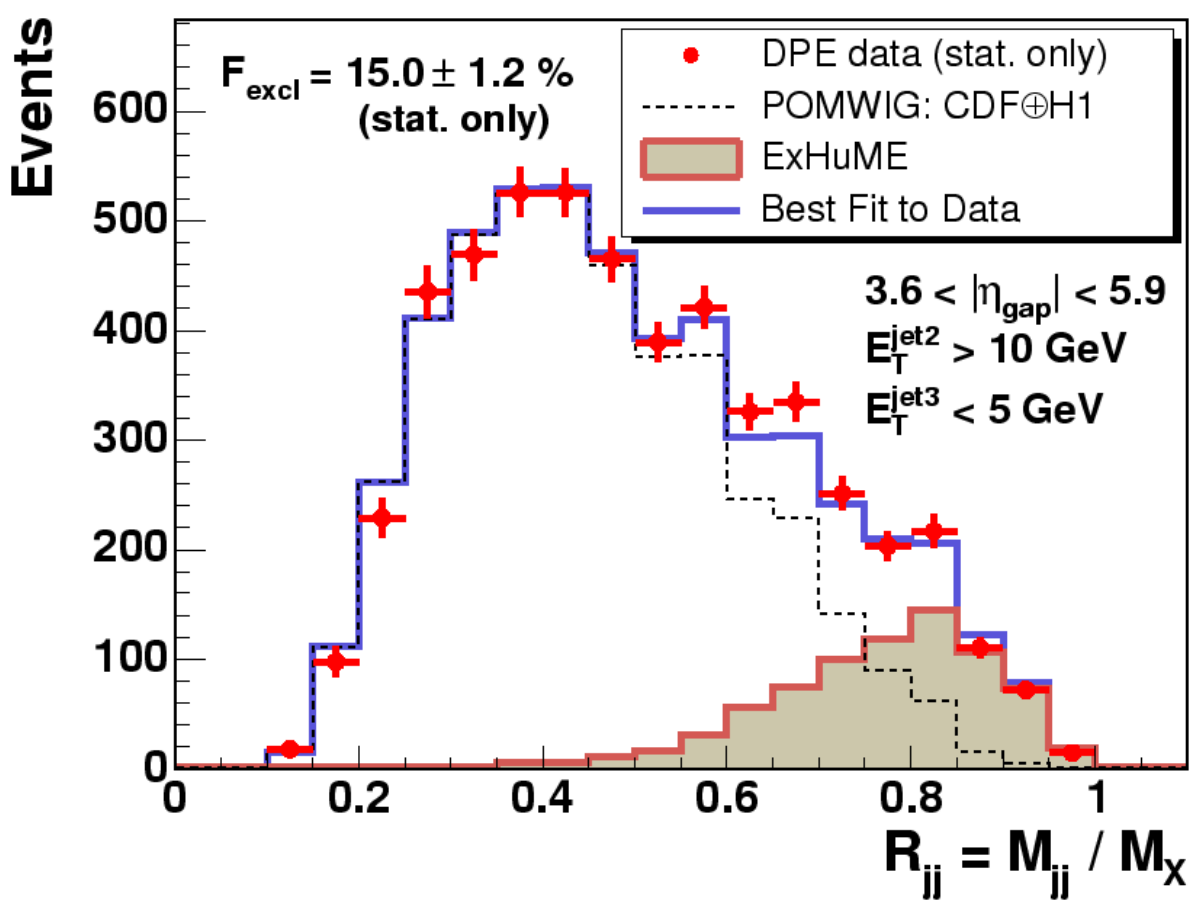

Many comparisons with varying $M C$ modelling and DPDFs ... ...hard to get rid of signal! Fit with free normalisation of inclusive, exclusive models to quantify exclusive part ... 


\section{CDF Exclusive Dijet Cross Section}

- ExHuME model based on KMR calculation ...

- $4.5 \%$ gap survival prob

- "Uncertainty factor 2.5"

- Expressed in terms of $M_{\mathrm{jj}}$,

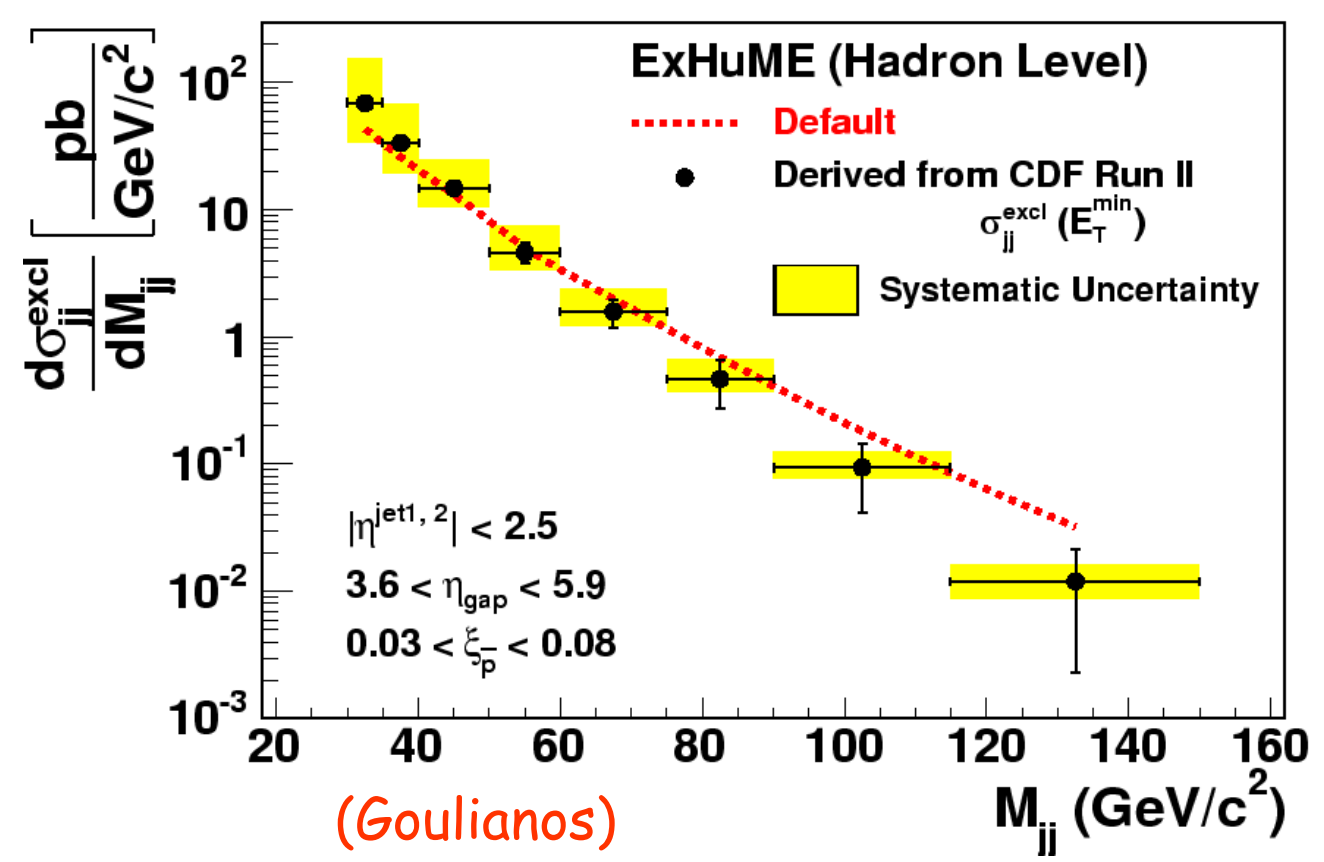

signal extends into possible Higgs discovery mass region!

\section{CDF Exclusive Di-photons}

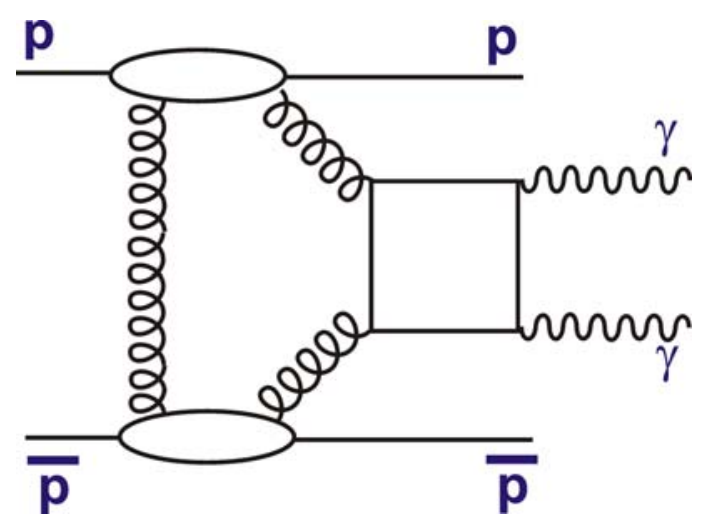

2/3 candidates with bkgrd $0.09+/-0.04$

KMR predicts 0.8 events (big uncty.)
(Pinfold)

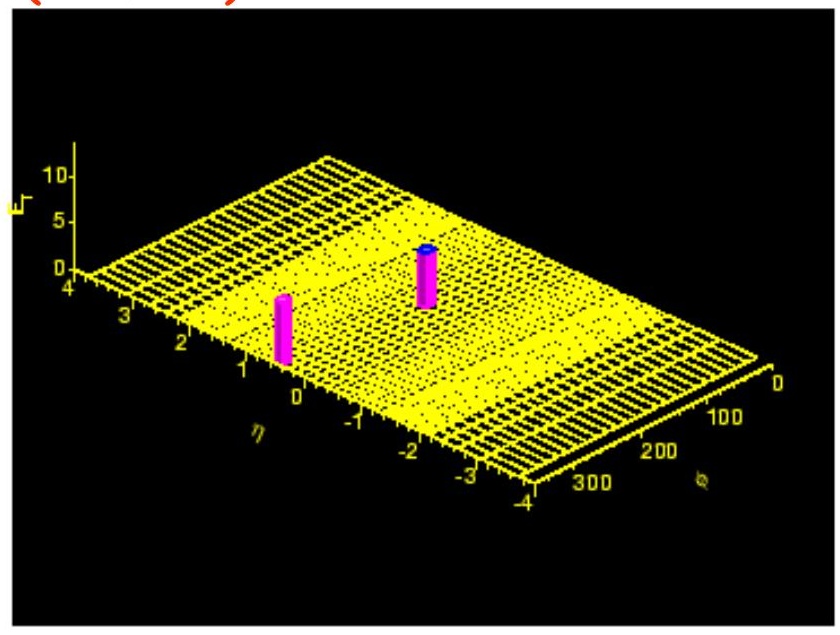


Some experimental successes of Workshop Understanding the dynamics of diffraction in detail ...

$-\mathrm{H} 1 \vee$ ZEUS $\mathrm{F}_{2} \mathrm{D}$ in much better agreement $\rightarrow$ DPDFs

- Dijet $\gamma p \rightarrow$ some understanding of $S^{2}$ for photons

- Connections between ep and pp via HERA-Tevatron

- New studies of $\gamma$ IP (and $\gamma \gamma$ and $\gamma p$...)

- Theory / expt input to LHC detector development

- New diffractive LHC programmes (e.g. ALICE)

... which tests (non-trivial) ingredients of LHC predictions

THANKS TO - ALL EXPERIMENTAL SPEAKERS IN 2006-2008 WORKSHOPS

- CO-CONVENORS FOR GOOD (EDUCATIONAL!) COLLABORATION 